\title{
An Analytical Solution of the Solution-Diffusion-Electromigration Equations Reproduces Trends in Ion Rejections during Nanofiltration of Mixed Electrolytes
}

\author{
Andriy Yaroshchuk ${ }^{\mathrm{a}, \mathrm{b}} *$ and Merlin L. Bruening ${ }^{\mathrm{c}, *}$ \\ a'ICREA, pg.L.Companys 23, 08010 Barcelona, Spain \\ ${ }^{b}$ Department of Chemical Engineering, Polytechnic University of Catalonia, av. Diagonal \\ 647,08028 Barcelona, Spain
}

'Departments of Chemical and Biological Engineering and Chemistry, University of Notre Dame, Notre Dame, IN 46556, USA

*corresponding author. E-mail: andriy.yaroshchuk@upc.edu; mbruenin@nd.edu

\begin{abstract}
This paper develops an analytical solution to the equations governing the solution-diffusionelectromigration transport of the ions from two salts that contain a common ion. The analytical expressions, which rely on constant ion permeances, readily enable simple spreadsheet computations of the permeate ion concentrations in nanofiltration (NF). Despite the model simplicity, calculations of ion rejections as a function of transmembrane volume fluxes, feed-solution compositions, and permeance values reproduce experimental trends, with ion permeances as the only adjustable parameters. For example, with solutions containing dissolved $M A$ and $M_{2} B$, when the membrane permeance to $B^{2-}$ is much lower than the permeances to $A^{-}$and $\mathrm{M}^{+}$, plots of $A^{-}$rejection versus transmembrane volume flux show the negative minimum that is often characteristic of NF. Moreover, $\mathrm{B}^{2-}$ rejection increases with increasing MA in the feed solution, reflecting a decreasing electric field in the membrane. The analytical solution also enables spreadsheet computations of electric fields in membrane separation layers and effectively models experimental data for intrinsic rejections as a function of transmembrane volume flow. Interestingly, the model suggests that with sufficiently high permeances of $A^{-}$(relative to $M^{+}$), at certain feed compositions the $A^{-} / B^{2-}$ selectivity may increase without bound as a function of volume flow. Otherwise, this selectivity does not vary greatly with feed composition and quickly reaches a limit as the volume flow increases. Despite the simplifying approximation of constant ion permeances, using only a few adjustable parameters this model readily reveals trends in NF ion rejections and may help to identify the desired ion permeances or solution compositions for specific separations.
\end{abstract}




\section{Keywords}

Nanofiltration, solution-diffusion, electromigration, negative rejection, ion permeance

\section{Introduction}

Nanofiltration (NF) is an effective, commercial method for water softening and salt purification. [1, 2] In contrast to reverse osmosis membranes, which reject nearly all salts in desalination, NF membranes selectively reject divalent ions and organic molecules with molecular weights $>150-500 \mathrm{Da}$. [1, 3] Moreover, NF membranes are more permeable to water than reverse osmosis membranes and thus require relatively low operating pressures if the osmotic pressure is not high.[3] However, ion rejections in selective NF membranes vary with solution composition, and rejections in single-salt solutions can differ greatly from those with salt mixtures.[4-8] Additionally, in experiments with salt mixtures containing ions whose permeances are very different, NF membranes often show negative ion rejections (higher ion concentrations in the permeate than in the feed) for the more permeable ions.[9-11] This paper develops the solution-diffusion-electromigration model to calculate ion rejections over a range of feed compositions and transmembrane volume fluxes. We provide an analytical solution to the differential equations that describe ion transport during NF of solutions containing two mixed salts with a common ion. As with other algebraic solutions of transport equations,[12] this analytical solution enables rapid exploration of trends in the behavior of membrane systems. The specific model assumes a solution-diffusion-electromigration mechanism with constant ion permeances and allows rapid spreadsheet calculations of individual ion fluxes to examine trends in selectivities and ion rejections as a function of ion permeances, feed solution compositions, and transmembrane volume fluxes. Additionally, we determine the conditions needed to observe large negative rejections.

Many models of NF membranes invoke nanoporous materials that exhibit hindered transport of ions along with steric, electric and dielectric exclusion.[13-20] These models adequately fit filtration data, but they require parameters such as volume charge and dielectric constant that vary with the feed solution for a given membrane.[6, 16, 21-23] Experimental assessment of the many variables in these complex models is a major challenge. Moreover, two recent studies suggest that the diffusion coefficients of dyes in nanoporous membranes are incompatible with the diameters of nanopores obtained from convective transport.[24, 25] Other work implies that convective ion transport in some NF membranes is negligible.[26] The very low salt permeances of membranes with ultrathin barrier layers [27-30] are also likely incompatible with the model of nanoporous membranes.

An alternative approach, which we employ in this work, describes ion transport in terms of phenomenological ionic permeances [26] and as a first approximation assumes that these permeances are independent of ion concentrations. Importantly, the use of single-ion permeances, rather than salt permeances, captures electromigration phenomena due to electrical potentials that arise from different permeances to cations and anions.[31, 32] Although this simple approach does not provide the mechanisms behind ion permeances, it allows modeling with only a few parameters that one may obtain through fitting of data. 
Additionally, it may provide previously unavailable input for comparison with mechanistic models.

Membrane permeances to individual ions are difficult to determine,[33] but large differences among the rejections of salts such as $\mathrm{NaCl}$ and $\mathrm{Na}_{2} \mathrm{SO}_{4}$ or $\mathrm{NaCl}$ and $\mathrm{MgCl}_{2}$ imply that for a given membrane the permeances to monovalent and divalent ions are very different.[4, 34, 35] For example, commercial $\mathrm{NF}$ membranes show $\mathrm{Na}_{2} \mathrm{SO}_{4}$ rejections around $99 \%$ and $\mathrm{NaCl}$ rejections of only 50-60\% in single-salt measurements.[36-38] Additionally, polyelectrolyte multilayer membranes exhibit highly selective transport of monovalent cations relative to divalent cations.[39-42] In electrodialysis with such membranes, $\mathrm{K}^{+} / \mathrm{Mg}^{2+}$ selectivities may be $>1000$.[42] These highly different permeances of monovalent and divalent ions should lead to unusual trends in NF ion rejections as a function of transmembrane water fluxes and feed compositions. Such trends are practically important because the pronounced NF selectivity between monovalent and multivalent ions leads to potential applications such as sulfate removal from chloralkali brines,[43] in-vacuum salt production[44] and metal/acid recovery.[45]

We should note that salt permeances can vary with concentration or composition, particularly for highly charged membranes. [26, 46-49] In studies of sulfonated polymers, Geise and coworkers showed that $\mathrm{NaCl}$ permeability increases with concentration, although $\mathrm{MgCl}_{2}$ permeability is relatively constant.[49-51] Nevertheless, a constant permeance can provide a reasonable first-order approximation of membrane performance,[32] particularly if feed solutions do not vary greatly. Additionally, for the model that we employ, permeances could depend on the feed concentration as long as the permeance across the membrane is a constant. This is similar to the Spiegler-Kedem model, which considers the solute permeability and reflection coefficient constant during the integration of transport equations across the membrane but has been frequently used to extract feed-concentration-dependent membrane parameters from experimental data.[34, 52,53] As long as the permeance is constant across the membrane, we can model rejection as a function of volume flow for a specific feed composition.

This study expands on our recently developed approach to the solution-diffusionelectromigration model [31] to provide an analytical solution to the constant-permeance model. The solution enables simple spread-sheet type calculations that reproduce trends in NF ion rejections from ternary electrolytes containing both monovalent and divalent ions. Analytical solutions are already available for modest rejections [11] or the cases of large and small transmembrane volume fluxes.[10, 54-56] However, those flux limits are often not accessible experimentally. In principle, our analysis applies to any flux, but we do neglect concentration polarization (CP) for simplicity. In interpretation of experiments one should correct for CP and obtain intrinsic rejections.[37, 57]

\section{Theory}

Eq(1) describes the flux, $j_{i}$, of ion $i$ through a membrane.

$-j_{i}=P_{i} \cdot\left(\frac{d c_{i}}{d x}+c_{i} Z_{i} \frac{d \varphi}{d x}\right)$ 
In this equation, $\varphi$ is the dimensionless (in $F / R T$ units) electrostatic potential in a virtual solution, $c_{i}$ is the concentration of ion $i$ in the virtual solution, $x$ is the transmembrane coordinate scaled on the membrane thickness, and $Z_{i}$ is the ion charge. The virtual (or reference) solution is a bulk electrolyte solution that could be in thermodynamic equilibrium with a given point inside the membrane. The virtual solution treatment is equivalent to the Nernst-Planck equation (see the supplementary material, SM), but because we use virtual concentrations, the permeances, $P_{i}$, include the partition coefficients. Thus, we do not need to separately specify partition and diffusion coefficients. Additionally, virtual solutions are electrically neutral, whereas solutions in a membrane may deviate from electroneutrality, especially if the membrane contains immobile charge or is ultrathin.[58]

For two salts with a common ion, $\mathrm{Eq}(1)$ is a system of three equations (one equation for each ion). At steady state $j_{i}$ is constant, and we assume constant values of $P_{i}$ throughout the membrane. Thus, the three equations represented in Eq(1) contain four variables, namely three virtual ion concentrations and the virtual potential. Because of electrical neutrality in the virtual solution, only two of the concentrations are independent, and we can solve the differential equations for the three independent variables. In principle, commercial software can readily solve this system of ordinary differential equations, but the iterative process of matching ion fluxes and permeate concentrations becomes multivariable in the case of electrolyte mixtures. Such a process is non-trivial because of the sensitivity to the choice of guess values and the possible existence of several local minima along with the true global minimum. The solution we present overcomes this challenge and uses analytical equations to calculate both permeate ion concentrations and volume flow as a function of a single variable, $u_{p}$, which is defined as twice the ratio of the ionic strength to total ion concentration in the permeate (see $\mathrm{Eq}(2)$ and Fig. 1 below). The treatment follows the general scheme we outlined previously,[31] but we specifically examine the case of three ions of different charges and give an explicit parametric analytical solution. The SM derives the solution, and we present the resulting equations below. Although the form of the solution appears complicated, one can readily program this solution into a spreadsheet to find permeate concentrations for given values of the feed concentration and flow rate. The SM gives a spreadsheet that implements the solution to $\mathrm{Eq}(1)$.

The solution to the equation relies on the definition of the relative double ionic strength, $u$,

$u=\frac{Z_{1}^{2} c_{1}+Z_{2}^{2} c_{2}+Z_{3}^{2} c_{3}}{c_{1}+c_{2}+c_{3}} \equiv \frac{2 I}{C}$

in $\mathrm{Eq}(2)$ where $C$ is the total ion concentration, $c_{1}+c_{2}+c_{3}$, and $2 I$ is the double ionic strength. The numeric subscripts refer to the different ions. Due to the electrical neutrality of the permeate solutions, one can express the permeate ion concentrations in terms of $u_{p}$ (the subscript $p$ refers to the permeate solution) and the total ion concentration in the permeate, $C_{p}$.

$c_{p 1}=C_{p} \cdot \frac{u_{p}+Z_{2} Z_{3}}{\left(Z_{1}-Z_{2}\right) \cdot\left(Z_{1}-Z_{3}\right)}$

$c_{p 2}=C_{p} \cdot \frac{u_{p}+Z_{1} Z_{3}}{\left(Z_{2}-Z_{1}\right) \cdot\left(Z_{2}-Z_{3}\right)}$

$c_{p 3}=C_{p} \cdot \frac{u_{p}+Z_{1} Z_{2}}{\left(Z_{3}-Z_{1}\right) \cdot\left(Z_{3}-Z_{2}\right)}$ 
Thus, an expression for $C_{p}$ as a function of $u_{p}$ will enable calculation of all of the ion concentrations in the permeate. $\mathrm{Eq}(4)$ gives such an expression, where the values of

$C_{p} \equiv C\left(u_{p}\right)=C_{0} \cdot\left[\frac{F_{m}\left(u_{p}\right)+u_{0}}{F_{m}\left(u_{p}\right)+u_{p}}\right]^{\frac{F_{m}\left(u_{p}\right)}{F_{p}\left(u_{p}\right)+F_{m}\left(u_{p}\right)}} \cdot\left[\frac{F_{p}\left(u_{p}\right)-u_{0}}{F_{p}\left(u_{p}\right)-u_{p}}\right]^{\frac{F_{p}\left(u_{p}\right)}{F\left(u_{p}\right)+F_{m}\left(u_{p}\right)}}$

of $F_{p}$ and $F_{m}$ depend only on $u_{p}$ and ion charges and permeances according to Eqs(5-8). The constants $u_{0}$ and $C_{0}$ in $\mathrm{Eq}(5)$ are the relative double ionic strength and total ion concentration in the feed solution, respectively, and we know these constants from specification of the feed composition.

$F_{p}\left(u_{p}\right) \equiv \frac{\sqrt{\left(\Pi \cdot \beta_{02}+\beta_{12} \cdot u_{p}\right)^{2}-4 \Pi \cdot\left(\Pi \cdot \beta_{01}+\beta_{11} \cdot u_{p}\right) \cdot\left(\Pi \cdot \beta_{-11}+\beta_{01} \cdot u_{p}\right)}-\left(\Pi \cdot \beta_{02}+\beta_{12} \cdot u_{p}\right)}{2 \cdot\left(\Pi \cdot \beta_{-11}+\beta_{01} \cdot u_{p}\right)}$

$F_{m}\left(u_{p}\right) \equiv \frac{\sqrt{\left(\Pi \cdot \beta_{02}+\beta_{12} \cdot u_{p}\right)^{2}-4 \Pi \cdot\left(\Pi \cdot \beta_{01}+\beta_{11} \cdot u_{p}\right) \cdot\left(\Pi \cdot \beta_{-11}+\beta_{01} \cdot u_{p}\right)}+\left(\Pi \cdot \beta_{02}+\beta_{12} \cdot u_{p}\right)}{2 \cdot\left(\Pi \cdot \beta_{-11}+\beta_{01} \cdot u_{p}\right)}$

$\beta_{i j} \equiv \frac{Z_{1}^{i} \cdot\left(z_{2}^{j}-Z_{3}^{j}\right)}{P_{1}}-\frac{Z_{2}^{i} \cdot\left(z_{1}^{j}-Z_{3}^{j}\right)}{P_{2}}+\frac{Z_{3}^{i} \cdot\left(Z_{1}^{j}-Z_{2}^{j}\right)}{P_{3}}$

$\Pi \equiv Z_{1} Z_{2} Z_{3}$

$\Sigma \equiv Z_{1}+Z_{2}+Z_{3}$

Finally, $\mathrm{Eq}(9)$ gives an expression for the volume flux, $J_{v}$, as a function of $u_{p}$ to allow correlation of $J_{v}$ with permeate ion concentrations or ion rejections.

$J_{v}\left(u_{p}\right)=\left(\frac{C_{0}}{C\left(u_{p}\right)}-1\right) \cdot \frac{\left(Z_{1}-Z_{2}\right)\left(Z_{2}-Z_{3}\right)\left(Z_{1}-Z_{3}\right)}{\Pi \cdot \beta_{-11}+\beta_{01} \cdot u_{p}}$

Eqs $(4,9)$ provide a parametric relationship between the transmembrane volume flux and the total ion concentration in the permeate, $C_{p}$, as functions of $u_{p}$. We then use $\mathrm{Eq}(3)$ to calculate the permeate concentrations of specific ions for the given values of $u_{p}$ and $C_{p}$.

Summary of the Calculation Procedure. From values for the charges and permeances of the three ions, we first calculate the needed values of $\beta_{i j}$, $\Pi$, and $\Sigma$ using Eqs(6-8). The specified feed composition allows computation of $u_{0}$ with $\mathrm{Eq}(2)$. Subsequently, for a range of values of $u_{p}$, we calculate the constants $F_{p}\left(u_{p}\right)$ and $F_{m}\left(u_{p}\right)$ from Eq(5). Finally, the $F_{p}\left(u_{p}\right)$ and $F_{m}\left(u_{p}\right)$ values enable determination of $C\left(u_{p}\right)$ from $\mathrm{Eq}(4)$, and we compute $J_{v}\left(u_{p}\right)$ from $\mathrm{Eq}(9)$.

Concentrations of the specific ions in the permeate then follow from $\mathrm{Eq}(3)$. For calculations of permeate ion concentrations as a function of the feed composition at a fixed $J_{v}$ value, we repeat the procedure for each feed composition, using $\mathrm{Eq}(9)$ to iteratively determine the value of $u_{p}$ that corresponds to $J_{v}$. With a composition-dependent $u_{p}\left(J_{v}\right)$, for each feed solution one can compute $C\left(u_{p}\right)$ from $\mathrm{Eq}(4)$ and specific ion concentrations from $\mathrm{Eq}(3)$. The Excel ${ }^{\mathrm{TM}}$ file in the SM gives an example of using these equations to calculate ion rejections as functions of volume flux and feed composition. 
Selectivity and the Range of $u_{p}$ Values. For definiteness, below we consider the ions " 1 " and " 2 " as anions and the common ion " 3 " as a cation. We will also consider the charge of ion " 2 " as larger than the charge of ion " 1 ". As an example, in a solution containing salts $M A$ and $M_{2} B$, ions " 1 ", " 2 " and " 3 " correspond to $\mathrm{A}^{-}, \mathrm{B}^{2-}$, and $\mathrm{M}^{+}$, respectively. Using $\mathrm{Eq}(3)$ and the corresponding equations for the feed solution ( $\mathrm{Eq}(\mathrm{S} 26), \mathrm{SM})$, one can define the ion selectivity, $S_{12}$, for ion " 1 " over ion "2"

$S_{12} \equiv \frac{c_{p 1} / c_{01}}{c_{p 2} / c_{02}}=\frac{u_{p}+Z_{2} Z_{3}}{u_{0}+Z_{2} Z_{3}} \cdot \frac{u_{0}+Z_{1} Z_{3}}{u_{p}+Z_{1} Z_{3}}$

To effectively use the parametric relationship of $\operatorname{Eqs}(4,9)$, we should determine the range of values that $u_{p}$ may take. Fig. 1 shows the physically possible values of $u$ for any solution containing the salts $\mathrm{MA}$ and $\mathrm{M}_{2} \mathrm{~B}$. In addition to giving the range of $u$, the figure shows the relationship between $u$ and a variable with a more intuitive meaning, $\chi_{M A}$, the salt mole fraction of $M A$ in a solution containing $M A$ and $M_{2} B$. For solutions of $M A$ and $M_{2} B, u$ ranges from a value of 2 when no $M A$ is present to 1 when $M_{2} B$ is absent. However, depending on the ion permeances and initial solution compositions, varying the transmembrane volume flux, $J_{v}$, will not give permeates whose compositions cover the entire range from $\chi_{M A}=0$ to $\chi_{M A}=1$. For example, if the feed solution contains both $M A$ and $M_{2} B$, and the permeance of $B^{2-}$ is low compared to the permeances of other ions, the permeate will never contain a smaller salt fraction of $\mathrm{MA}$ than the feed. Additionally, unlimited $\mathrm{A}^{-} / \mathrm{B}^{2-}$ selectivities are only possible for certain ranges of feed compositions and ion permeances. Next we develop explicit

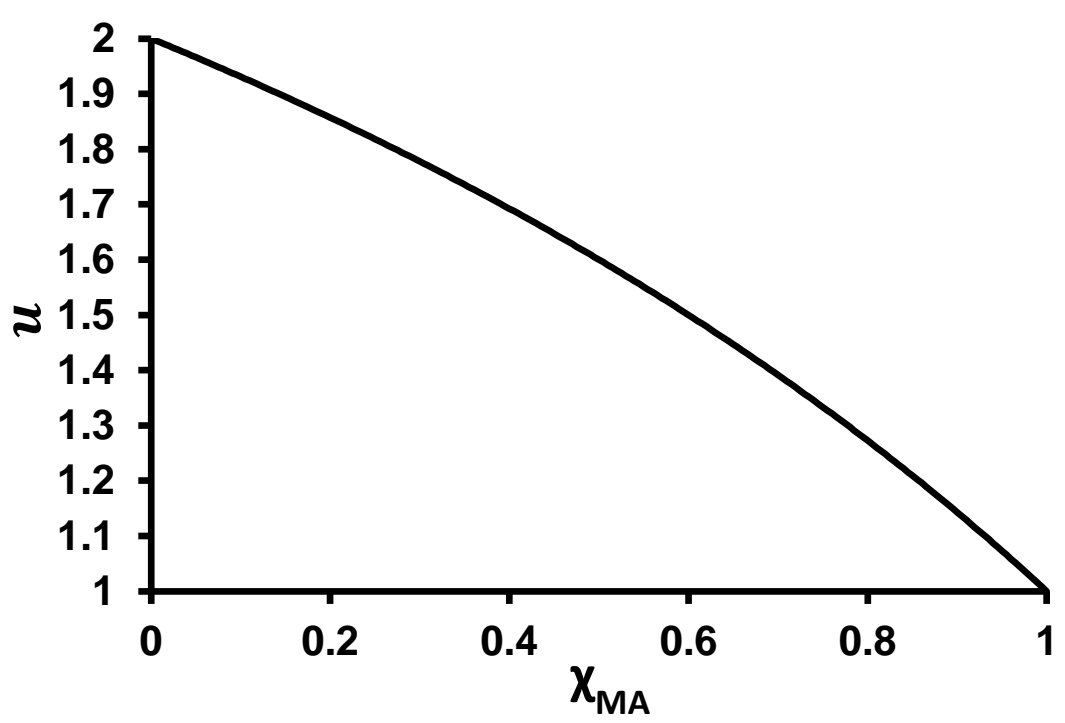

Fig. 1. Values of the relative double ionic strength, $u$ (see $\mathrm{Eq}(2)$ ), as a function the mole fraction of $M A, \chi_{M A}$, in a solution containing the salts $M A$ and $M_{2} B$ where $A^{-}$and $B^{2-}$ are the salt anions. More precisely, $\chi_{M A}=[M A] /\left([M A]+\left[M_{2} B\right]\right)$.

expressions for the range of possible $u_{p}$ values. 
At very small transmembrane volume fluxes, the solution composition in the permeate is the same as in the feed, i.e. the membrane approaches equilibrium and the compositions on both sides are nearly the same. Therefore, $u_{p} \rightarrow u_{0}$. When the permeance to anion " 2 " (e.g. $\left.\mathrm{B}^{2-}\right)$ is less than to anion " 1 " (e.g. $\left.\mathrm{A}^{-}\right), u_{0}$ is the maximum value for $u_{p}$. According to $\mathrm{Eq}(9)$, for very large transmembrane volume fluxes the total ion concentration in the permeate, $C\left(u_{p}\right)$, must tend to zero (below we will see that the second factor in the right-hand side of Eq(9) remains finite at any physically meaningful value of $u_{p}$ ).

Based on $\mathrm{Eq}(4)$, the total ion concentration in the permeate should approach zero when either

$F_{p}\left(u_{p}\right)-u_{0} \rightarrow 0$

or

$F_{m}\left(u_{p}\right)+u_{p} \rightarrow 0$

Eq (11) makes the second term in brackets in $\mathrm{Eq}(4)$ go to zero. Because of the negative exponent of the first term in brackets in $\mathrm{Eq}(4)$, the denominator in this bracketed term must go to zero for $C\left(u_{p}\right)$ to approach zero, and this gives $\operatorname{Eq}(12)$. From $\operatorname{Eqs}(11,5)$, we obtain the minimum value of $u_{p}, u_{p m i n}$, which occurs when $C_{p} \rightarrow 0$ (see the SM for details of this

$u_{p \min }=-\Pi \cdot \frac{u_{0}^{2} \cdot \beta_{-11}+u_{0} \cdot \beta_{02}+\Pi \cdot \beta_{01}}{u_{0}^{2} \cdot \beta_{01}+u_{0} \cdot \beta_{12}+\Pi \cdot \beta_{11}}$

solution). Depending on the permeances of the three ions, $\left(u_{p m i n}+Z_{1} Z_{3}\right)$ will either be positive for all physical values of $u_{0}$ (see below) or turn negative for a certain range of feed compositions. A negative value of $\left(u_{\text {pmin }}+Z_{1} Z_{3}\right)$ implies negative concentrations of ion " 2 " in the permeate (see $\mathrm{Eq}(3)$ ), so for such situations $\mathrm{Eq}(11)$ does not apply, and one should use $\mathrm{Eq}(12)$ to determine the value of $u_{\text {pmin }}$. Solving Eq(12) gives Eq(14) (see the SM).

$u_{p \min }=-Z_{1} Z_{3}$

Eqs $(14,3)$ imply that at this $u_{\text {pmin }}$ the permeate contains no anion " 2 ". Below, we will see that the values of the ion permeances determine whether $\mathrm{Eq}(11)$ or $\mathrm{Eq}(12)$ applies to a given system. Physically, for some permeance values and feed concentrations, the permeate can never become pure MA and contain no anion " 2 " $\left(\mathrm{B}^{2-}\right)$. In such situations, $\mathrm{Eq}(11)$ applies. The transition between the conditions for Eqs(11 and 12) yields a qualitative difference in iontransport selectivity as a function of transmembrane volume flux. Fig. 2 shows some examples of $C_{p}\left(u_{p}\right)$ calculated using Eq(4). Indeed, depending on the ionic permeances and feed composition (see below for more details) $C_{p}$ tends to zero either at values of $u_{p}>-Z_{1} Z_{3}$ or at $u_{p}=-Z_{1} Z_{3}$. For this simulation $-Z_{1} Z_{3}=1$.

We have considered the case of two anions and a common cation. For mixtures with two cations and a common anion, Eqs $(11,12)$ change to

$F_{m}\left(u_{p}\right)+u_{0} \rightarrow 0$

and 
$F_{p}\left(u_{p}\right)-u_{p} \rightarrow 0$

These changes occur because the exponents in $\mathrm{Eq}(4)$ have different signs on going from a common cation to a common anion. Otherwise, the behavior remains the same.

(a)

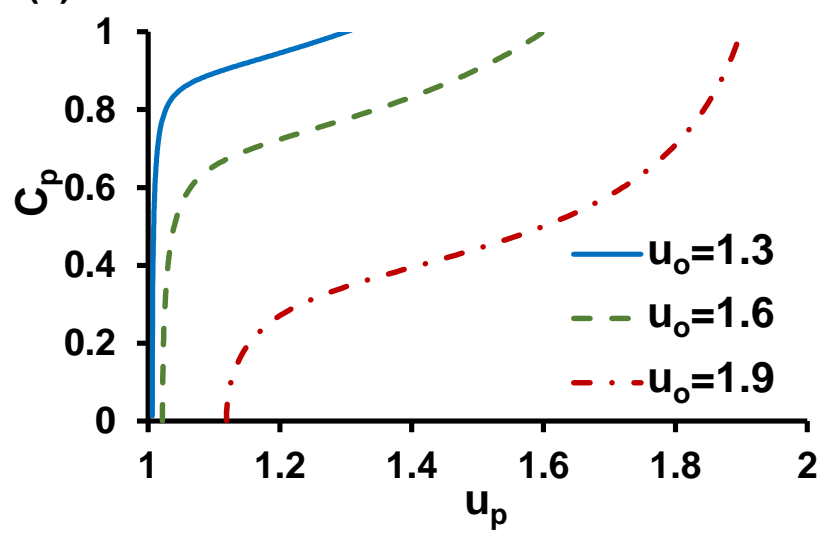

(b)

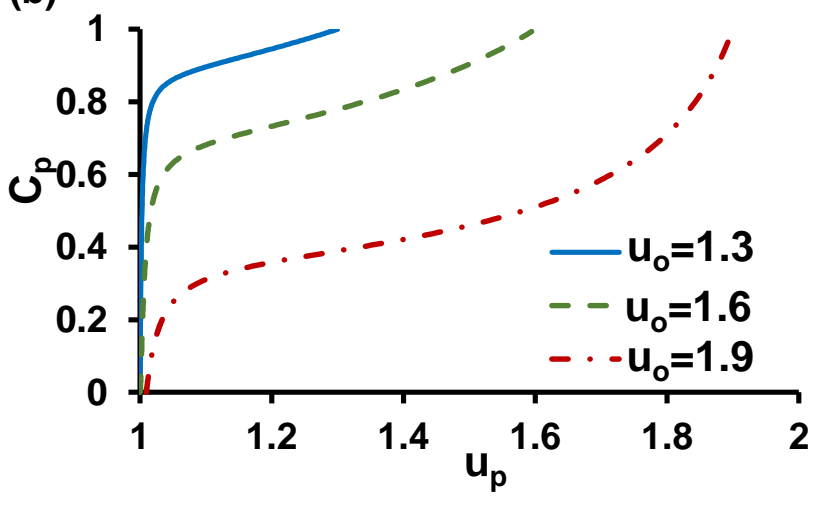

(c)

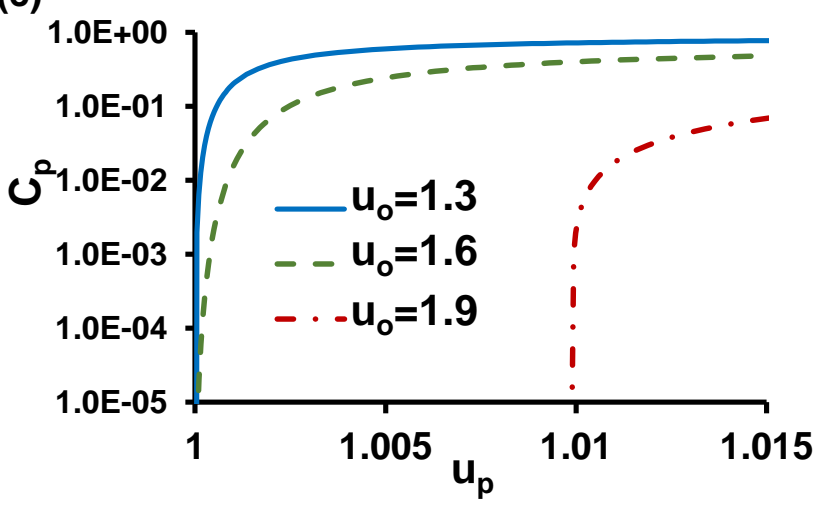

Fig. 2. Calculated total ion concentrations in the permeate, $C_{p}$, as a function of $u_{p}$ and $u_{0}$. In this example $C_{0}=1$, and $u_{0}$ values of $1.3,1.6$, and 1.9 correspond to $\chi_{\mathrm{MA}}$ values of $0.778,0.500$, and 0.143 , respectively, where the ions " 1 ", " 2 ", and " 3 " are $\mathrm{A}^{\prime}, \mathrm{B}^{2-}$, and $\mathrm{M}^{+}$, respectively. The permeances are: (a) $P_{A^{-}}=1 \mu \mathrm{m} / \mathrm{s} ; P_{B^{2-}}=0.01 \mu \mathrm{m} / \mathrm{s} ; P_{M^{+}}=10 \mu \mathrm{m} / \mathrm{s}$ and (b) $P_{A^{-}}=$ $10 \mu \mathrm{m} / \mathrm{s} ; P_{B^{2-}}=0.01 \mu \mathrm{m} / \mathrm{s} ; P_{M^{+}}=1 \mu \mathrm{m} / \mathrm{s}$. Figure (c) enlarges the x-axis of Figure (b) at low values of $u_{p}$. Note that the minimum value of $u_{p}$ depends on the values of both $u_{0}$ and ion permeances. 


\section{Results and Discussion}

This section first discusses the limiting case of single salts and shows that the 3 -ion model is consistent with the solution-diffusion model for transport of a single salt. Subsequently, we show how salt rejections and ion-transport selectivities vary with the transmembrane volume flux and solution composition, and discuss calculated electric fields and modeling of some specific literature experimental data. Finally, we describe the conditions that lead to strong, observable negative rejections in NF.

\section{Limiting case of single salts}

In the limiting case of a single-salt, the 3-ion transport model presented here should simplify to the typical solution-diffusion model, $[59,60]$ in which $\mathrm{Eq}(17)$ relates the salt concentration in the permeate to the transmembrane volume flux.

$c_{p}=\frac{j_{i}}{J_{v}}=\frac{P_{s}\left(c_{0}-c_{p}\right)}{J_{v}}$

In this equation, $P_{s}$ is the salt permeance of the membrane, and $c_{0}$ and $c_{p}$ are salt concentrations in the feed and permeate, respectively. Rearrangement of Eq(17) gives

$J_{v}=\left(\frac{c_{0}}{c_{p}}-1\right) P_{s}$

Eqs $(9,18)$ have similar forms, but in $\mathrm{Eq}(9)$ the coefficient analogous to $P_{S}$ is not constant but a function of $u_{p}$, which depends on the permeate composition. In the limiting cases of either single salt (e.g. a solution containing only $M A$ or only $M_{2} B$ ), the parameter $u$ (see $E q(2)$ ) changes neither across the membrane nor with the transmembrane volume flux and is

$u=-Z_{i} Z_{3}$

where $i=1$ or $i=2$. Using the definitions of $\beta_{i j}$ in $\mathrm{Eq}(6)$, and substituting $\mathrm{Eq}(19)$ for $u_{p}$ into the part of $\mathrm{Eq}(9)$ that is analogous to $P_{S}$ (left-hand side of $\mathrm{Eq}(20)$ ), one can show that

$\frac{\left(Z_{1}-Z_{2}\right)\left(Z_{2}-Z_{3}\right)\left(Z_{1}-Z_{3}\right)}{\Pi \cdot \beta_{-11}+\beta_{01} \cdot u_{p}}=\frac{\left(Z_{i}-Z_{3}\right) \cdot P_{i} \cdot P_{3}}{Z_{i} P_{i}-Z_{3} P_{3}}=P_{S}$

The middle expression in $\mathrm{Eq}(20)$ is the definition of the salt permeance, $P_{S}$, to either of two single salts consisting of ions " 1 " and " 3 " or " 2 " and " 3 ". Thus, Eq(9) satisfies the limiting cases for either single salt as it should.

The expression on the left-hand side of Eq(20) depends monotonically on $u_{p}$, and the two possible values for the middle of $\mathrm{Eq}(20)$ correspond to substitution of $u_{p}$ for either of the two single salts. Moreover, the values of $u_{p}$ for these salts are the upper and lower bounds of physical $u_{p}$ values (see Fig. 1). Thus, the left-hand side of $\mathrm{Eq}(20)$ is finite. Looking at Eq(9), this implies that as the transmembrane volume flux tends toward infinity, the total ion concentration in the permeate must tend to zero as expected in a solution diffusion model and as we assumed in the Theory section when defining the range of $u_{p}$ values. 


\section{Rejections as a function of transmembrane volume flux in the three-ion system}

As Eq(18) shows, in single-salt experiments the concentration of salt in the permeate decreases with increasing volume flux, $J_{v}$, so salt rejection increases with increasing $J_{v}$. Eq(21) defines the rejection, $R_{i}$, of a specific ion.

$R_{i}=1-\frac{c_{p i}}{c_{0 i}}$

For the single-salt case, the rejections of the cation and anion are equal. In contrast, with a 3ion system, the ratios of ions in the permeate can vary while still maintaining electroneutrality, and specific ion rejections need not increase monotonically with $J_{v}$.

Fig. 3 shows calculated ion rejections as a function of $J_{v}$ for a solution containing equimolar $M A$ and $M_{2} B\left(X_{M A}=0.5\right)$. (For the notation in this paper this equimolar solution corresponds to $u_{0}=1.6$, and $\mathrm{A}^{\prime}, \mathrm{B}^{2-}$, and $\mathrm{M}^{2+}$ are ions " 1 ", " 2 ", and " 3 ", respectively.) In Fig. 3, the divalent ion $\mathrm{B}^{2-}$ has the lowest permeance of the ions and hence shows the highest rejection, which increases monotonically with the transmembrane volume flux. The rejection of the monovalent anion, $A^{-}$, is negative at low volume fluxes but eventually turns positive as the flow increases. In this case, negative rejection stems from spontaneously arising electric fields that accelerate anions and decelerate cations to give zero net current. This field appears predominantly because of the low permeance to $\mathrm{B}^{2-}$, so it is especially pronounced when the feed fraction of monovalent anions is low. Nonetheless, Fig. 3 shows that significant negative rejections occur even at a 1:1 MA: $\mathrm{M}_{2} B$ feed composition $\left(u_{0}=1.6\right)$. Monovalent anion
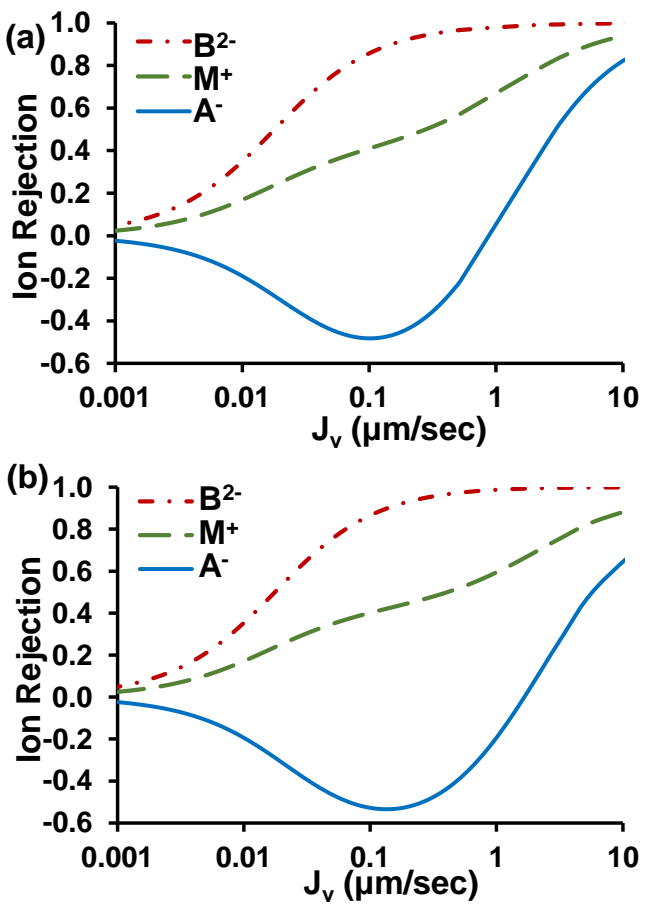

Fig. 3. Calculated ion rejections vs. transmembrane volume flux, $J_{v}$, for a solution containing equimolar $\mathrm{MA}$ and $\mathrm{M}_{2} \mathrm{~B}$ salts dissolved in the feed solution. The assumed ionic permeances (in $\mu \mathrm{m} / \mathrm{s}$ ) are (a) $P_{A^{-}}=1 ; P_{B^{2-}}=0.01 ; P_{M^{+}}=10$; and (b) $P_{A^{-}}=10 ; P_{B^{2-}}=0.01 ; P_{M^{+}}=1$. The equimolar, $\chi_{\mathrm{MA}}=0.5$, solution corresponds to $u_{0}=1.6$, and the log scale on the $\mathrm{x}$-axis improves the visibility of differences in the range where the transmembrane volume flux is low. Dashed-dot red lines, dashed green lines, and solid blue lines correspond to rejection of $\mathrm{B}^{2-}, \mathrm{M}^{+}$ and $\mathrm{A}^{-}$, respectively. 
rejections initially become more negative with increasing transmembrane volume flux because faster flow decreases the concentrations of $\mathrm{B}^{2-}$ and $\mathrm{M}^{+}$in the permeate. These lower permeate concentrations give rise to higher concentration gradients for diffusion of $\mathrm{M}^{+}$and $\mathrm{B}^{2-}$ and, hence, larger electric fields that accelerate $\mathrm{A}^{-}$and $\mathrm{B}^{2-}$ and decelerate $\mathrm{M}^{+}$to maintain zero current. As $J_{v}$ initially increases, the electrical migration of $\mathrm{A}^{-}$(toward the permeate) increases more rapidly than $J_{v}$ increases, so the rejection becomes more negative. However, eventually $J_{v}$ increases more rapidly than the electrical migration of $A^{-}$, and dilution of the permeate leads to positive rejections.

The rejection of $\mathrm{M}^{+}$lies between the rejections of the two anions and gets closer to the rejection of $A^{-}$with increasing volume flux because the rejection of the doubly charged anion approaches $100 \%$. When the permeate contains minimal $\mathrm{B}^{2-}$, the concentrations of $\mathrm{A}^{-}$and $\mathrm{M}^{+}$ in the permeate are essentially equal, but their rejections are not equal because their feed concentrations differ.

Figs. 3(a) and 3(b) show two distinct cases of lower and higher permeance for the monovalent anion $\left(P_{A^{-}}\right)$relative to the permeance of the common monovalent cation $\left(P_{M^{+}}\right)$. There are no clearly discernible qualitative differences between these two cases. Nevertheless, below we show that this is due to the poor visibility of differences in ion rejections when they approach $100 \%$. In terms of ion selectivity, the difference between these two cases is much more visible.

\section{Ion Rejection versus Feed Solution Composition}

In some situations, the feed composition dramatically affects the electric fields that spontaneously arise in the membrane and thus greatly alters ion rejections. $[4,11]$ For a volume flux of $10 \mu \mathrm{m} / \mathrm{s}$, Fig. 4a shows how the rejection of $A^{-}, R_{A^{-}}$, varies with the feed mole fraction, $\chi_{M A}$, of $M A$ relative to the total dissolved salt, i.e. $\chi_{M A}=[M A] /\left([M A]+\left[M_{2} B\right]\right)$. When $P_{A^{-}}$is 10 times the permeance of $\mathrm{M}^{+}$(solid blue line, Fig. 4a), $R_{A^{-}}$decreases with the addition of $M_{2} B$ (decreasing $\chi_{M A}$ ) and eventually becomes negative when $\chi_{M A}$ is about 0.1 . This is consistent with NF data that frequently show negative rejections of trace monovalent ions.[11, 37] In contrast, if $P_{M^{+}}$is 10 times $P_{A^{-}}, R_{A^{-}}$is nearly constant over a wide range of solution compositions (dashed red line, Fig. 4a). This essentially constant $R_{A^{-}}$implies that the spontaneously arising electric field does not vary greatly with solution composition when $P_{M^{+}}$ is an order of magnitude greater than $P_{A^{-}}$. With a relatively high $P_{M^{+}}$value, the electric field maintains zero electric current primarily through deceleration of $\mathrm{M}^{+}$, regardless of whether $\mathrm{A}^{-}$ or $\mathrm{B}^{2-}$ is the dominant anion (see the scheme in Fig. $5 \mathrm{a}$ ). Thus, at the relatively high volume flux and rejections in Fig. 4a (dashed red line), the electric field that overcomes most of the diffusive $\mathrm{M}^{+}$transport does not vary strongly with solution composition, so $R_{A^{-}}$is nearly constant. On the other hand, when $P_{A^{-}}$is an order of magnitude greater than $P_{M^{+}}$, at high values of $\chi_{\text {MA }}$ the electric field maintains zero electric current primarily by decelerating $A^{-}$(see Fig. 5b). However, as $\chi_{M A}$ declines and $B^{2-}$ becomes the dominant ion, the electric field will decrease and eventually change sign to decelerate $\mathrm{M}^{+}$and accelerate anions (Fig. $5 \mathrm{c}$ ). The decrease and eventual change in the direction of the electric field leads to diminishing and eventually negative rejection of $A^{-}$as $\chi_{M A}$ decreases (Fig. 4a, blue line). 

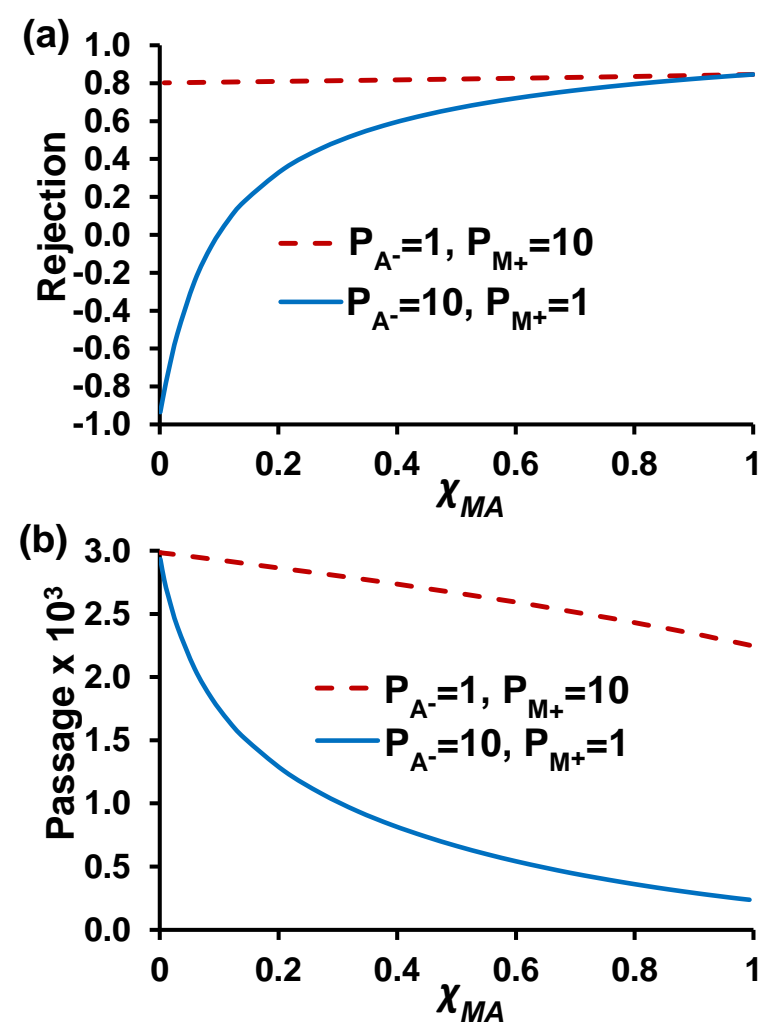

Fig.4. Calculated (a) rejections of $A^{-}$and (b) passages of $B^{2-}$ versus the mole fraction, $\chi_{M A}$, of salt that is $\mathrm{MA}$ in a solution containing $\mathrm{MA}$ and $\mathrm{M}_{2} \mathrm{~B}$. The calculation employs $J_{v}=10 \mu \mathrm{m} / \mathrm{s}$ and ionic permeances (in $\mu \mathrm{m} / \mathrm{s}$ ) of $P_{M^{+}}=1 ; P_{A^{-}}=10 ; P_{B^{2-}}=0.01$ (blue lines) or $P_{M^{+}}=10 ; P_{A^{-}}=1 ; P_{B^{2-}}=0.01$ (dashed red lines).

(a)

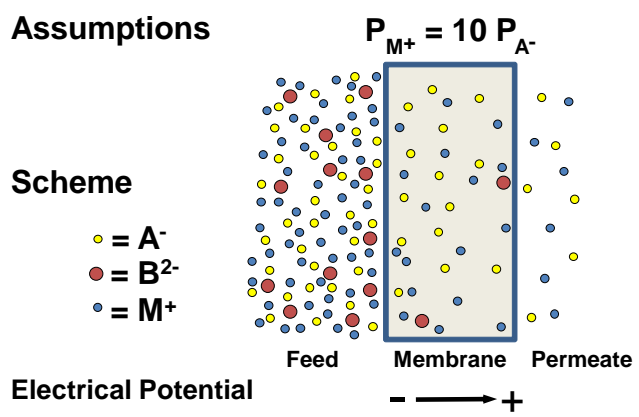

\section{Ion Fluxes}

Diffusion $\rightarrow$
Electro-
migration

Net flux $\rightarrow$ (b)
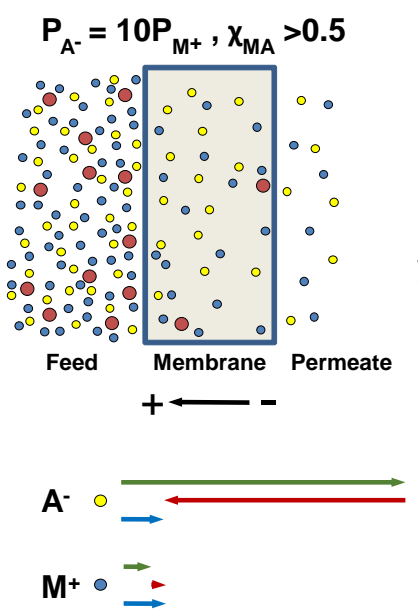

(c)
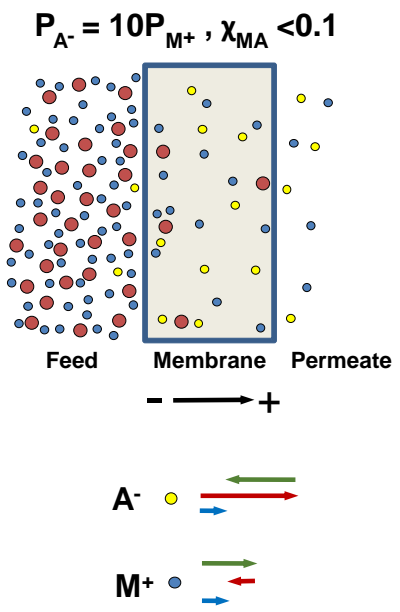

Fig. 5. Qualitative diagrams of ion transport, electrical potentials, and ion fluxes (diffusion, electromigration, and net) as a function of ion permeances and salt mole fractions, $\chi_{M A}$, in the feed. In all cases, $P_{B^{2-}}$ is low compared to the permeances of $\mathrm{A}^{-}$and $\mathrm{M}^{+}$, so the flux of $\mathrm{B}^{2-}$ is negligible. Lengths of green, red and blue arrow qualitatively represent fluxes.

Due to the low permeance that we assumed for $\mathrm{B}^{2-}$, its rejection remains above 0.997 
regardless of solution composition at a volume flux of $10 \mu \mathrm{m} / \mathrm{s}$. However, a plot of (1rejection), or ion passage, clearly shows that $\mathrm{B}^{2-}$ passage decreases (rejection increases) for solutions containing an increasing fraction of MA (Fig. 4b). The effect is more pronounced when $P_{A^{-}}=10 \mu \mathrm{m} / \mathrm{s}$ and $P_{M^{+}}=1 \mu \mathrm{m} / \mathrm{s}$ than when $P_{A^{-}}=1 \mu \mathrm{m} / \mathrm{s}$ and $P_{M^{+}}=10 \mu \mathrm{m} / \mathrm{s}$. The decrease in the passage of a divalent ion with the addition of a monovalent salt is often, though not always, a feature of NF.[61-64] In the absence of MA, the electric field achieves zero electric current by decelerating $\mathrm{M}^{+}$(large effect) and accelerating $\mathrm{B}^{2-}$ (small effect due to the low $P_{B^{2-}}$ ). With the addition of $\mathrm{MA}$, the field can decelerate additional $\mathrm{M}^{+}$and accelerate the more permeable $A$ to achieve zero current with a smaller electric field (e.g. Fig. 5c). The decrease in electric field decreases the passage of $B^{2-}$ due to reduced electromigration of this ion. When $\mathrm{A}^{-}$is less permeable than $\mathrm{M}^{+}$, the addition of $\mathrm{MA}$ has less of an effect on the transport of $\mathrm{B}^{2-}$. For the permeances we chose, $R_{B^{2-}}$ is always high, but with higher values of $P_{B^{2-}}$, rejection of $\mathrm{B}^{2-}$ will decrease and changes in $R_{B^{2-}}$ will become more apparent. In water softening, the rejection of divalent ions will generally increase as the concentration of monovalent salts increases.

Rejections of $\mathrm{M}^{+}$(not shown) decrease monotonically with increasing $\chi_{\mathrm{MA}}$ because of the low permeance of $B^{2-}$ relative to $A^{-}$. The initial decrease as $\chi_{M A}$ increases is most dramatic with a high value of $P_{A^{-}}$. At $\chi_{\mathrm{MA}}=0$ and $\chi_{\mathrm{MA}}=1, R_{M^{+}}$is equal to $R_{B^{2-}}$ and $R_{A^{-}}$, respectively.

As discussed above, trends in the rejection of individual ions as a function of feed composition depend on the relative permeances of the monovalent anion and the common monovalent cation (Fig. 4). However, according to $\mathrm{Eq}(20)$ and Fig. 4a, the rejection of MA salt when $\chi_{M A}=1$ (no $M_{2} \mathrm{~B}$ ) is the same for $P_{A^{-}}=10 \mu \mathrm{m} / \mathrm{s}, P_{M^{+}}=1 \mu \mathrm{m} / \mathrm{s}$ and $P_{M^{+}}=10, P_{A^{-}}=1 \mu \mathrm{m} / \mathrm{s}$. This occurs because we simply switched the permeances of $\mathrm{M}^{+}$and $\mathrm{A}^{-}$. The $\mathrm{M}_{2} B$ rejection when $\chi_{\mathrm{MA}}=0$ (no MA) is also essentially the same for $P_{A^{-}}=10 \mu \mathrm{m} / \mathrm{s}, P_{M^{+}}=1 \mu \mathrm{m} / \mathrm{s}$ and $P_{M^{+}}=10$, $P_{A^{-}}=1 \mu \mathrm{m} / \mathrm{s}$ because of the very low value of $P_{B^{2-}}$. Thus, ion rejections for the two limiting cases of single salts are insensitive to whether the monovalent cation or the monovalent anion has the order of magnitude greater permeance. In contrast, in solutions that contain mixed salts ion rejections vary greatly depending on the relative permeances of the monovalent anion and common monovalent cation.

We should emphasize that the calculations in this section assume that permeance does not vary with feed composition. A previous study with NF270 membranes revealed only small $(<50 \%)$ variations in the permeances of $\mathrm{Na}^{+}$and $\mathrm{Cl}^{-}$in solutions containing $\mathrm{NaCl}, \mathrm{MgSO}_{4}$, $\mathrm{Na}_{2} \mathrm{SO}_{4}$, and $\mathrm{MgCl}_{2}$ as the dominant salts. [37] Sulfate showed low permeances in solutions of several different salts. Thus, the assumption of constant permeance should approximate ion rejections well in that case. However, for other ions such as $\mathrm{Mg}^{2+}$ and other membranes or widely varying salt concentrations, permeances may show greater variation with feed composition.[37, 49, 65] 
Calculation of Electric Fields and Concentration Profiles in the Membrane Selective Layer

The previous section showed that trends in rejection as a function of feed concentration often reflect the electric fields that arise spontaneously during transport. Section S3 of the SM describes how to compute electric fields and concentration profiles as a function of $u_{p}$, and Fig. 6 shows the electric fields calculated for the conditions depicted in Fig. 5. To transform to a dimensional electric field, one should multiply the dimensionless field by $R T$ / FL where $L$ is the membrane thickness. For a selective layer thickness of $25 \mathrm{~nm}, R T / F L$ is $10^{6} \mathrm{~V} / \mathrm{m}$, so the dimensional fields are in the range of megavolts per meter. Additionally, the field varies greatly across the membrane and is a strongly non-linear function of coordinate. Therefore, the

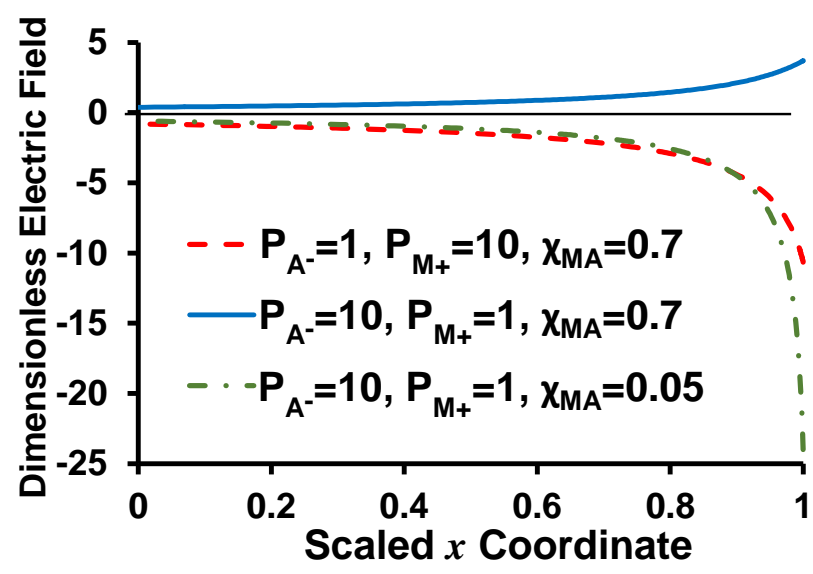

Fig. 6. Calculated dimensionless electric fields as a function of scaled distance into the membrane selective layer. The calculation employs $J_{v}=10 \mu \mathrm{m} / \mathrm{s}$ and permeances (in $\mu \mathrm{m} / \mathrm{s}$ ) as indicated in the legend. The permeance of $\mathrm{B}^{2-}$ was $0.01 \mu \mathrm{m} / \mathrm{s}$, and the figure indicates the salt mole fraction of $M A$, defined as $[M A] /\left([M A]+\left[M_{2} B\right]\right)$. The $\chi_{M A}$ values of 0.7 and 0.05 correspond to $u_{0}$ values of 1.391 and 1.966 , respectively.

approximation of constant electric field clearly does not apply to our system. The signs of the electric fields in Fig. 6 are consistent with the electrical potentials depicted in Fig. 5, remembering that the electric field is the negative of the spatial derivative of the electrical potential.

In most cases, the virtual concentration profiles in the separation layer are essentially linear, which is consistent with an electric field that increases greatly near the permeate side to maintain a constant electromigration flux in this region despite low ion concentrations. For ions with negative rejections, however, the concentration profile is not linear and shows a minimum somewhere in the selective layer.

\section{Modeling Literature Nanofiltration Data}

This section explores modeling of literature data with the analytical solution for 3-ion transport. Fig. 7 shows reported rejections of $\mathrm{Na}^{+}, \mathrm{Cl}^{-}$, and $\mathrm{Ca}^{2+}$ ions during nanofiltration of a solution containing dissolved $\mathrm{NaCl}$ and $\mathrm{CaCl}_{2}$.[66] These data are corrected for concentration polarization to represent real (intrinsic) rejections. To fit the data, we first obtained the average $\mathrm{CaCl}_{2}$ permeance from a single-salt literature experiment that measured $\mathrm{CaCl}_{2}$ rejections (corrected for concentration polarization) as a function of volume flux.[66] The combination of this $\mathrm{CaCl}_{2}$ permeance, $\mathrm{Eq}(20)$, and a guess value for the $\mathrm{Cl}^{-}$permeance gives a 


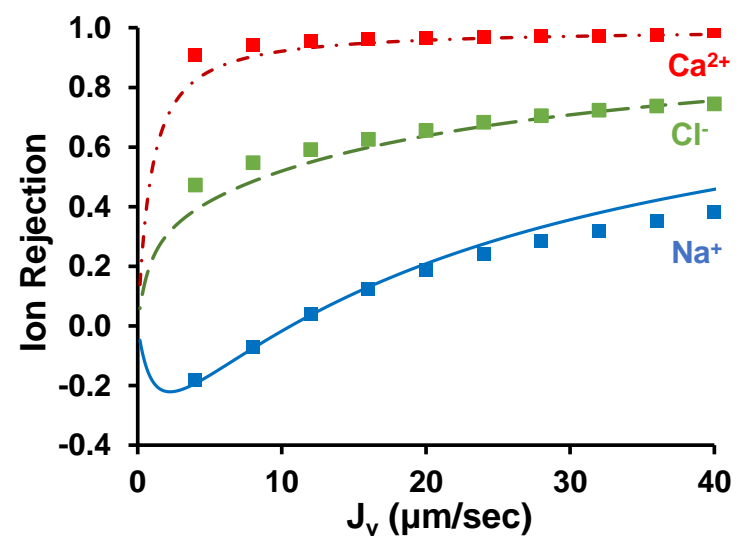

Fig. 7. Experimental (squares) and simulated (lines) real ion rejections during nanofiltration of a solution containing $0.0286 \mathrm{M} \mathrm{CaCl}_{2}$ and $0.429 \mathrm{M} \mathrm{NaCl}\left(0.667\right.$ ratio of $\mathrm{Ca}^{+}$to $\left.\mathrm{Na}^{2+}\right)$ through a Desal DK membrane. The literature data come from Figure $8 \mathrm{~b}$ in reference [66], and the simulation employs ion permeances of $0.57 \mu \mathrm{m} / \mathrm{s}$ for $\mathrm{Ca}^{2+}$ and $24 \mu \mathrm{m} / \mathrm{s}$ for $\mathrm{Cl}^{-}$and $\mathrm{Na}^{+}$.

value for the $\mathrm{Ca}^{2+}$ permeance. Assuming equal permeances for $\mathrm{Cl}^{-}$and $\mathrm{Na}^{2+}$, we then calculated rejections of the three ions as a function of volumetric flux using $\operatorname{Eqs}(4,9,2)$. We varied the value of $\mathrm{Cl}^{-}$permeance until the calculated data showed the best visual agreement with the trends in literature data (Fig. 7). Thus, the fit to the data employs only one adjustable parameter because we assumed equal $\mathrm{Cl}^{-}$and $\mathrm{Na}^{2+}$ permeances. The calculated and experimental rejections differ by less than 0.1 , although at the lower values of $J_{v}$ in Fig. 7 the differences increase between the calculated and experimental intrinsic rejections for $\mathrm{Ca}^{2+}$ and $\mathrm{Cl}^{-}$. This may result from the challenge in correcting the literature data for concentration polarization, as even with the single-salt data, the $\mathrm{CaCl}_{2}$ permeance approximately doubles on going from the lowest to the highest volume flux (see Table S1). Fig. S2 in the SM shows the fitting of data at different ratios of $\mathrm{Ca}^{2+}$ to $\mathrm{Na}^{+}$in the feed solution. Higher ratios of $\mathrm{Ca}^{2+}$ to $\mathrm{Na}^{+}$ require lower values of the $\mathrm{Cl}^{-}$and $\mathrm{Na}^{+}$permeances to fit the data, which could stem from $\mathrm{Ca}^{2+}$ adsorption that decreases the membrane permeability. Nevertheless, when the $\mathrm{Ca}^{2+}$ to $\mathrm{Na}^{+}$ ratio varies from 0.67 to 4 , the values of fitted $\mathrm{Cl}^{-}$and $\mathrm{Na}^{+}$permeances only change from 24 to 20.

Fig. S3a (SM) shows modeling of a set of data for nanofiltration of a solution containing dissolved $\mathrm{NaCl}$ and $\mathrm{MgCl}_{2}$.[67] The fit is similar to that in Fig. 7, showing that the model gives reasonable agreement for two different systems. In the modeling of Fig. S3, we had to use two permeances as fitting parameters because $\mathrm{MgCl}_{2}$ single-salt rejections are anomalously low (See Table S2 for values). Nevertheless, variation of the $\mathrm{Mg}^{2+}$ permeance has only a minor effect on the fitted value for the $\mathrm{Cl}^{-}$and $\mathrm{Na}^{+}$permeances. Thus, fitting of two permeances is feasible. We should note, however, that from these data we cannot determine separate values of $\mathrm{Cl}^{-}$and $\mathrm{Na}^{+}$permeances, as varying these parameters independently does not yield obviously better fits to the data (see Figure S3b).

\section{Ion Selectivity vs Ionic Permeances}

As mentioned in the theory section, for some combinations of ion permeances and feed compositions $u_{p}$ cannot take the full range of values $\left(-Z_{1} Z_{3}\right.$ to $\left.-Z_{2} Z_{3}\right)$ specified by two permeates that each contain only one of the two anions (see Fig. 2). This section demonstrates that these changes in the behavior of $u_{p \min }$ as a function of $u_{0}$ correspond to 
qualitative changes in the variation of selectivity vs. transmembrane volume flux. The definition of ion selectivity ( $\mathrm{Eq}(10))$ shows that when $u_{p} \rightarrow-Z_{1} Z_{3}$ the selectivity for ion " 1 " over "ion " 2 " increases without bound as the transmembrane volume flux increases. In contrast, for systems where $u_{p} \rightarrow u_{p \min }>-Z_{1} Z_{3}$, the limiting selectivity as $J_{v} \rightarrow \infty$ is finite. We can detect these two kinds of behavior using $\mathrm{Eq}(13)$ and considering if $\left(u_{p \min }+Z_{1} Z_{3}\right)$ becomes negative for physically-meaningful values of $u_{0}$. Specifically, from Eq(13) one can show (see the SM for details) that $\left(u_{p m i n}+Z_{1} Z_{3}\right)$ can take negative values when

$\frac{P_{1}}{P_{3}}>\frac{Z_{2}-Z_{3}}{Z_{2}-Z_{1}}$

Notably, the criterion of $\mathrm{Eq}(22)$ does not depend on the permeance to ion " 2 ". If $\mathrm{Eq}(22)$ is satisfied, within a part of the range of $u_{0}$ (feed composition), the minimum value of relative double ionic strength in the permeate, $u_{p m i n}$, defined by Eq(13) becomes smaller than the physical limit set by Eq(14). This occurs when

$-Z_{1} Z_{3}<u_{0}<-\Pi \cdot \frac{P_{1}-P_{3}}{Z_{1} P_{1}-Z_{3} P_{3}}$

Outside the range of $\mathrm{Eq}(23)$ and the condition specified in $\mathrm{Eq}(22), u_{p m i n}>-Z_{1} Z_{3}$ so $\mathrm{Eq}(13)$ defines the lower limit of variation of $u_{p}$, and the ion selectivity remains finite. If the condition of $\mathrm{Eq}(22)$ is not satisfied, ion selectivity remains finite at any feed composition.

Fig. 8 illustrates these trends. In Fig. 8 a the calculations use a $P_{1} / P_{3}$ (equivalently $P_{A^{-}} / P_{M^{+}}$) value that does not satisfy $\mathrm{Eq}(22)$ so selectivity depends only modestly on the feed composition and quickly tends to saturation with increasing transmembrane volume flux. In contrast, the $P_{1} / P_{3}$ value for Fig. 7b satisfies Eq(22), and the ion selectivity depends more on the feed composition. The selectivity changes from unlimited to limited when the feed
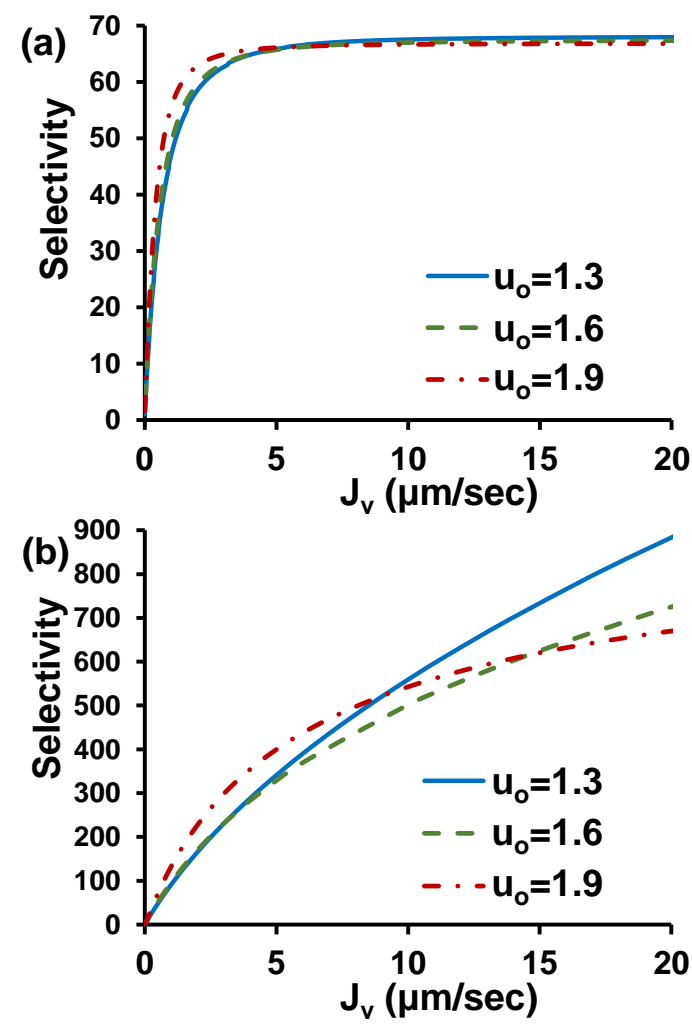

Fig. 8. Calculated ion selectivities, $\mathrm{A}^{-} / \mathrm{B}^{2-}$, as a function of transmembrane volume flux, $J_{v}$, and feed solution composition (represented by $u_{0}$ ). In this calculation $u_{0}$ values of $1.3,1.6$, and 1.9 correspond to $\chi_{\mathrm{MA}}$ values of $0.778,0.5$, and 0.143 . The permeances are: (a) $P_{A^{-}}=$ $1 \mu \mathrm{m} / \mathrm{s} ; P_{B^{2-}}=0.01 \mu \mathrm{m} / \mathrm{s} ; P_{M^{+}}=10 \mu \mathrm{m} / \mathrm{s}$ and (b) $P_{A^{-}}=10 \mu \mathrm{m} / \mathrm{s} ; P_{B^{2-}}=0.01 \mu \mathrm{m} / \mathrm{s} ; P_{M^{+}}=1 \mu \mathrm{m} / \mathrm{s}$. 
composition changes from $u_{0}=1.6$ to $u_{0}=1.9$. Using Eq(23) one can show that for the ion permeances in Fig.3b the qualitative change of behavior occurs at $u_{0}=1.64$.

Qualitatively we can understand these trends based on the electric fields that spontaneously arise due to the differences in membrane permeances to various ions (see Fig. 6 for some examples). These electric fields increase with increasing volume fluxes because the ion concentrations decrease in the permeate and diffusive flux increases. We consider first the case where $P_{M^{+}}>P_{A^{-}}$(Fig. 8a), and the spontaneous electric field accelerates $\mathrm{A}^{-}$and $\mathrm{B}^{2-}$. These conditions do not satisfy $\mathrm{Eq}(22)$. As $J_{v}$ increases from zero, initially selectivity increases because the permeate concentrations depart more and more from the feed concentrations, and $P_{A^{-}}$is higher than $P_{B^{2-}}$. Once the permeate concentrations are small compared to the feed concentrations, the electric field and the selectivity are essentially constant. Note that the selectivity is less than $P_{A^{-}} / P_{B^{2-}}$ because the charges on the ions are not the same and the ratio of electromigration fluxes of the two ions is not just the ratio of their permeances.

When $P_{A^{-}}>P_{M^{+}}$(Fig. 8b) and the feed mole fraction of $\mathrm{B}^{2-}$ is not too high $\left(u_{0}\right.$ is low), the spontaneously arising electric field retards both $A^{-}$and $B^{2-}$. This considerably increases the rejection of anion $\mathrm{B}^{2-}$ in particular due to its larger charge. Thus, as $J_{v}$ increases and diffusion increases, the increasing electric field affects the flux of $B^{2-}$ more than the flux of anion $A^{-}$. Even at fairly large $J_{v}$ values, small increases in the electric field decrease the flux of $\mathrm{B}^{2-}$ to near zero values to increase selectivity. However, as the feed mole fraction of $\mathrm{B}^{2-}$ increases $\left(u_{0}\right.$ increases), the difference in the membrane permeances to $\mathrm{B}^{2-}$ and $\mathrm{M}^{+}$will eventually control the spontaneously arising electric field, which will change direction to accelerate anions and decelerate cations. Accordingly, the field will then lower the rejection of both $\mathrm{B}^{2-}$ and $\mathrm{A}^{-}$. Because electrical migration and diffusion are both proportional to permeance, the selectivity becomes essentially constant when the volume flow is large.

The above discussion shows that the ion selectivity behaves in fundamentally different ways depending on whether $P_{A^{-}} / P_{M^{+}}$is sufficiently high to satisfy Eq(22). Although the model is phenomenological and does not specify the mechanisms of relationships between the ionic permeances, it is useful to briefly consider examples where $\mathrm{Eq}(22)$ should or should not apply. We recently developed a model where unequal anion and cation partitioning into ultra-thin membrane barrier layers leads to significant space-charge regions in the barrier.[58] This model postulates that due to differences among ions in their excess solvation energies, selective ion dissolution in the barrier leads to charge separation and electrical potential gradients that tend to equalize partition coefficients. For example, if due to excess solvation energies a barrier layer excludes doubly charged anions more than monovalent cations (this assumption agrees with lower permeances to doubly charged anions), the inter-phase electrostatic potential will attract anions to and repel cations from the barrier layer. As a result, the partitioning of monovalent anions and cations will increase and decrease, respectively, relative to the hypothetical situation of intrinsic partitioning due to the excess solvation energies of monovalent ions alone. If additionally the excess solvation energies are approximately equal for the monovalent cations and anions, the inter-phase potential (due to exclusion of the doubly charged anions) will make the partition coefficient of monovalent anions larger than that of monovalent cations. For a sufficiently high electrostatic potential, this effect might make the membrane permeance to the monovalent anions large enough to 
satisfy the condition of Eq(22). In this case, the monovalent/divalent anion selectivity could continually increase with increasing volume flows.

In contrast, a negative surface charge or a larger intrinsic excess solvation energy for monovalent anions than monovalent cations will lead to higher permeances for the monovalent cation relative to the monovalent anion. If these factors dominate, the condition of Eq(22) will not occur and selectivity over a divalent anion will remain finite as $J_{v}$ increases. In summary, depending on the barrier-layer properties either of the two situations in Fig. 8 may occur. With typical negatively charged commercial NF membranes, the condition of $\mathrm{Eq}(22)$ (or its counterpart for two salts with a common anion) is more likely to occur for mixtures with doubly charged cations than with doubly charged anions.

\section{Negative rejections of ions}

Negative rejection of specific ions is a signature feature of NF of multi-ion solutions. Fig. 9 shows some examples of particularly large calculated negative rejections. Two important parameters characterize the negative rejection: the location $U_{v}$ value) of the minimum and its depth.

$\mathrm{Eq}(24)$ defines the condition for the location of the minimum, which occurs at the maximum permeate concentration of ion " 1 ", or A".

$\frac{d c_{p 1}\left(u_{p}\right)}{d J_{v}}=0$

Using $\mathrm{Eq}(9)$, one can show that

$\frac{d J_{v}\left(u_{p}\right)}{d u_{p}} \neq \infty$

Therefore, the criterion of the minimum is also

$\frac{d c_{p 1}\left(u_{p}\right)}{d u_{p}}=0$

Using $\mathrm{Eq}(3)$, we can further specify the criterion of $\mathrm{Eq}(26)$ as

$\frac{d \ln C_{p}}{d u_{p}}+\frac{1}{u_{p}+Z_{2} Z_{3}}=0$

Finally, using Eq(4) we obtain

$\frac{d \ln C_{p}}{d u_{p}}=\frac{F_{p} F_{m}^{\prime}-F_{m} F_{p}^{\prime}}{\left(F_{p}+F_{m}\right)^{2}} \cdot \ln \left(\frac{F_{m}+u_{0}}{F_{m}+u_{p}} \cdot \frac{F_{p}-u_{p}}{F_{p}-u_{0}}\right)+\frac{u_{0}-u_{p}}{F_{p}+F_{m}} \cdot\left[\frac{F_{p} F_{p}^{\prime}}{\left(F_{p}-u_{p}\right)\left(F_{p}-u_{0}\right)}-\frac{F_{m} F_{m}^{\prime}}{\left(F_{m}+u_{p}\right)\left(F_{m}+u_{0}\right)}\right]+$
$\frac{u_{p}}{\left(F_{p}-u_{p}\right)\left(F_{m}+u_{p}\right)}$

where we have denoted

$F_{p, m}^{\prime} \equiv \frac{d F_{p, m}\left(u_{p}\right)}{d u_{p}}$ 
$F_{p}$ and $F_{m}$ are algebraic functions of $u_{p}$. Therefore, one can further expand $\mathrm{Eq}(28)$ by using the definitions of Eqs(5-8) but the resulting expression is cumbersome so we do not include it here. In any case, the equation for the dimensionless permeate relative double ionic strength that corresponds to the strongest negative rejection is transcendental and requires numerical solution. To obtain the location (transmembrane volume flux) and the depth of the minimum we substitute the value of $u_{p}$ found from $\operatorname{Eqs}(27,28)$ into $\operatorname{Eqs}(3,4,9)$.
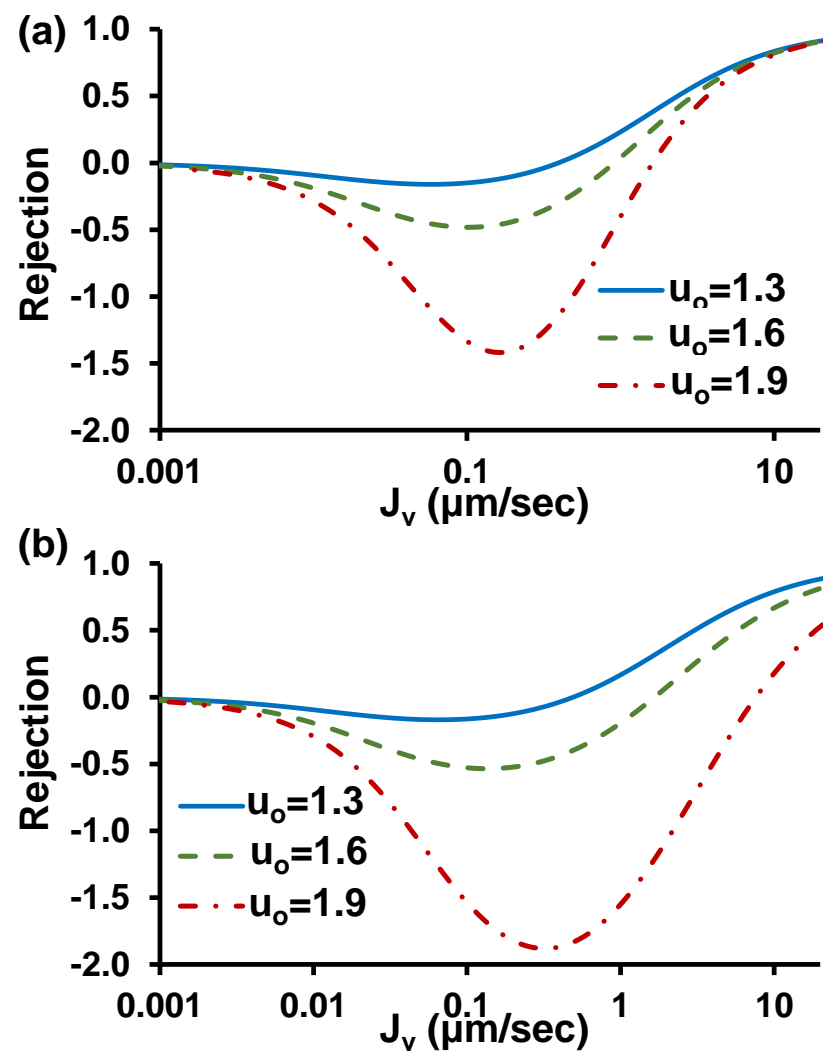

Fig.9. Calculated $A^{-}$rejections vs. transmembrane volume flux, $J_{v}$, for a solution containing MA and $\mathrm{M}_{2} \mathrm{~B}$ salts in the feed solution. The assumed ionic permeances (in $\mu \mathrm{m} / \mathrm{s}$ ) are (a) $P_{A^{-}}=$ $1 ; P_{B^{2-}}=0.01 ; P_{M^{+}}=10$; and (b) $P_{A^{-}}=10 ; P_{B^{2-}}=0.01 ; P_{M^{+}}=1$. The indicated $u_{0}$ values of $1.3,1.6$, and 1.9 correpond to correspond to $\chi_{\mathrm{MA}}$ values of $0.778,0.5$, and 0.143 .

Negative rejections usually occur at low transmembrane volume fluxes (see Fig.9), which makes experimental observation of their minima difficult. Frequently, one only observes the ascending branch to the right of the minimum . Nevertheless, sometimes the minima are detectable.[37]

Fig. 10 provides some insight into the minima "observability". When $P_{B^{2-}}\left(P_{2}\right)$ is very low, the minima are deep (Figs. 10c and 10d), but they appear at very small volume fluxes, especially when the feed mole fraction of $\mathrm{M}_{2} \mathrm{~B}$ is not too large (i.e. when $u_{0}$ approaches 1 , see Figs. 10a and 10b). At relatively large $P_{B^{2-}}$, the minima appear at "measurable" volume fluxes but are shallow. The best "observability" (sufficiently deep minima located at workable transmembrane volume fluxes) occurs at the intermediate value of $P_{2}=0.01 \mu \mathrm{m} / \mathrm{s}$ especially in the case $P_{A^{-}}>P_{M^{+}}$. This agrees with the experimental observation of a minimum for traces of $\mathrm{Na}^{+}$added to dominant $\mathrm{MgCl}_{2}$,[37] which should occur with a moderately low permeance to 
$\mathrm{Mg}^{2+}$ in combination with a high permeance to $\mathrm{Na}^{+}$and $\mathrm{PCl}_{\mathrm{Cl}^{-}}<\mathrm{P}_{\mathrm{Na}^{+}}$. However, the same study observed only the ascending branch for experiments with traces of $\mathrm{Cl}^{-}$added to $\mathrm{Na}_{2} \mathrm{SO}_{4}$ and $\mathrm{MgSO}_{4}$ (primarily due to the very low membrane permeance to $\mathrm{SO}_{4}{ }^{2-}$ ). Interestingly, for relatively large $P_{2}$ at sufficiently small feed mole fractions of doubly charged anions, negative rejections do not occur. This corresponds to the volume flux of the minimum going to zero at some $u_{0}>-Z_{1} Z_{3}$.
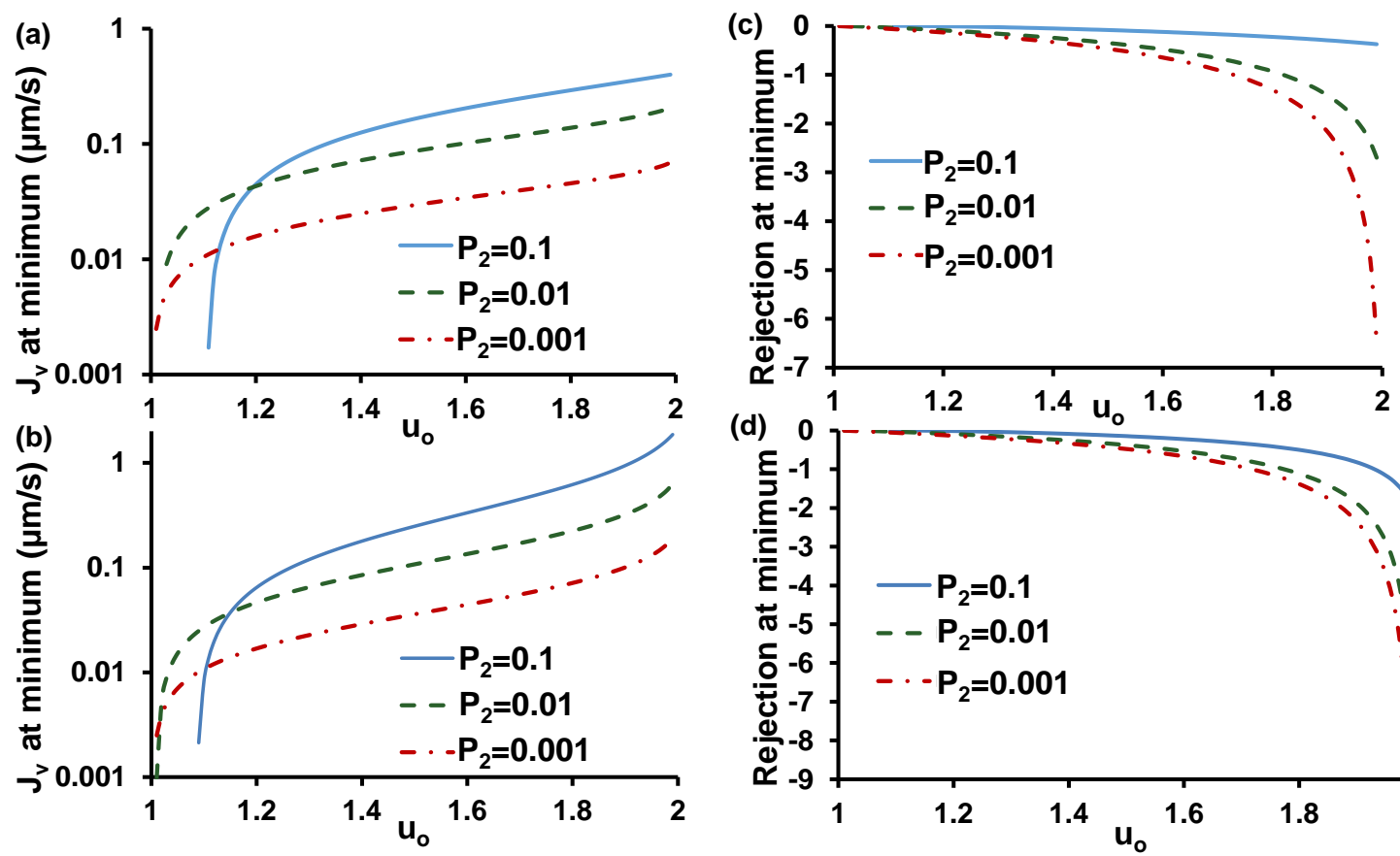

Fig.10. Calculated transmembrane volume flux at the minimum of negative rejection of ion $A^{-}$ $(a, b)$ and rejection at the point of minimum $(c, d)$ during filtration of a solution containing MA and $\mathrm{M}_{2} \mathrm{~B}$ at the feed compositions indicated by $u_{0}$. For this calculation: $P_{A^{-}}=1 \mu \mathrm{m} / \mathrm{s} ; P_{M^{+}}=$ $10 \mu \mathrm{m} / \mathrm{s}(\mathrm{a}, \mathrm{c}) ; P_{A^{-}}=10 \mu \mathrm{m} / \mathrm{s} ; P_{M^{+}}=1 \mu \mathrm{m} / \mathrm{s}(\mathrm{b}, \mathrm{d}) ;$ permeances to $\mathrm{B}^{2-}$ are indicated on the graphs as $P_{2}$.

The feed fraction of $\mathrm{B}^{2-}$ is also an important parameter. Expectedly, the minimum location shifts towards higher values of transmembrane volume flux when this fraction increases and, especially, when the $\mathrm{B}^{2-}$ fraction approaches $100 \%\left(u_{0}=2\right)$. Minima appear at especially large volume fluxes when the permeance to $A^{-}$is high and the permeance to $\mathrm{M}^{+}$is relatively low. In this case the monovalent anions make a significant contribution to the membrane electrical conductance even when their fraction is relatively small. This reduces the spontaneously arising transmembrane electric fields and the negative rejections caused by them. When this contribution vanishes at small feed fractions of monovalent anions, there is a sharp increase in the magnitude of negative rejections and their occurrence moves to larger volume fluxes. 


\section{Conclusions}

This paper develops an analytical solution to the differential equations that govern the coupled transport of three ions through NF membranes. This solution enables the calculation of ion permeate concentrations with a simple spreadsheet, which in turn allows exploration of ion rejections over a wide range of volume fluxes, solution compositions, and permeance values. Calculated trends in ion rejections for solutions containing monovalent and divalent ions are consistent with NF results that show (a) increasing divalent ion rejection with the addition of a monovalent salt, (b) minima in the negative rejection of monovalent ions as a function of transmembrane volume flux, and (c) increasingly negative monovalent anion rejections as the ratio of monovalent to divalent anions decreases.

The approximation of constant permeances is vital to obtaining a simple analytical solution to the coupled transport equations for three ions and comparing transport under different conditions. However, there are certainly cases in which permeances will vary with solution composition. Electrostatic effects on ion partitioning, for example, depend on the ionic strength of the solution. Moreover, divalent ion adsorption, and hence membrane surface charge, may depend on the concentration of the divalent ions. Nevertheless, the constant permeance model is often a useful approximation and effectively captures many experimental trends typical for NF.[32] Additionally, one could allow ion permeances to depend on feed composition as long as they do not vary across the membrane for a specific experiment. Given the ease of calculating ion rejections with this simple model, we think it may aid in designing specific NF separations and explaining unusual trends in NF ion rejections.

\section{Acknowledgments}

A.Y. acknowledges support from the project "Valorization of by-product from industrial effluents: integration of membrane technologies in the separation, concentration and purification steps" (Waste2Product, CTM2014-57302-R) funded by the Ministry of Economy and Competitiveness (MINECO, Spain) and the Catalan government (project ref. 2014SGR050). A.Y. and M.L.B. gratefully acknowledge funding from the Division of Chemical Sciences, Geosciences, and Biosciences, Office of Basic Energy Sciences of the U.S. Department of energy through Grant DE-FG02-98ER14907. We thank Professor Sébastien Déon of Université de Franche-Comté for the real rejection data.

\section{Description of Symbols}

$c_{i} \quad$ concentration of ion $i$ in the virtual solution

$c_{o} \quad$ salt concentration in the feed solution

$c_{p} \quad$ salt concentration in the permeate solution

$c_{p i} \quad$ concentration of ion $i$ in the permeate solution

$c_{0 i} \quad$ concentration of ion $i$ in the feed solution

$C(x)$ sum of the concentrations of all ions in the virtual solution 
$C_{0} \quad$ sum of the concentrations of all ions in the feed solution

$C_{p} \quad$ sum of the concentrations of all ions in the permeate solution

E electric field in the membrane selective layer

$F_{m} \quad$ function defined in $\mathrm{Eq}(5)$ to develop a concise solution

$F_{p} \quad$ function defined in $\mathrm{Eq}(5)$ to develop a concise solution

$i \quad$ index representing a specific ion

$j_{i} \quad$ flux of ion $i$

$J_{v} \quad$ volume flux

$I(x) \quad$ ionic strength in the virtual solution

$P_{i} \quad$ membrane permeance to ion $i$

$P_{S} \quad$ salt permeance of the membrane

$R_{i} \quad$ membrane rejection of ion $i$

$S_{12} \quad$ transport selectivity for ion 1 over ion 2

$u \quad \frac{2 I}{C}$, double ionic strength divided by the total ion concentration, or relative double ionic strength

$u_{p} \quad$ value of relative double ionic strength in the permeate solution

$u_{\text {pmin }}$ smallest possible physical value of relative double ionic strength in the permeate solution

$u_{0} \quad$ value of relative double ionic strength in the feed solution

$x \quad$ dimensionless distance into the membrane barrier layer

$Z_{i} \quad$ charge of ion $i$

$\beta_{i j} \quad$ constants in $\mathrm{Eq}(6)$, defined for concisely presenting equations

$\Pi \quad Z_{1} Z_{2} Z_{3}$, product of the charges of the three ions

$\Sigma \quad Z_{1}+Z_{2}+Z_{3}$, sum of the charges on the three ions

$\varphi \quad$ virtual electrostatic potential in $F / R T$ units

$\chi_{M A}[M A] /\left([M A]+\left[M_{2} B\right]\right)$, salt mole fraction of $M A$ in a solution containing $M A$ and $M_{2} B$ 


\section{References}

[1] A.I. Schäfer, A.G. Fane, T.D. Waite, Nanofiltration - principles and applications, Elsevier, Oxford, UK, 2005, pp. 560.

[2] B. Van der Bruggen, M. Mänttäri, M. Nyström, Drawbacks of applying nanofiltration and how to avoid them: A review, Sep. Purif. Tech., 63 (2008) 251-263.

[3] A.W. Mohammad, Y.H. Teow, W.L. Ang, Y.T. Chung, D.L. Oatley-Radcliffe, N. Hilal, Nanofiltration membranes review: Recent advances and future prospects, Desalination, 356 (2015) 226-254.

[4] H.M. Krieg, S.J. Modise, K. Keizer, H.W.J.P. Neomagus, Salt rejection in nanofiltration for single and binary salt mixtures in view of sulphate removal, Desalination, 171 (2005) 205-215. [5] A. Pérez-González, R. Ibáñez, P. Gómez, A.M. Urtiaga, I. Ortiz, J.A. Irabien, Nanofiltration separation of polyvalent and monovalent anions in desalination brines, J. Membr. Sci., 473 (2015) 16-27.

[6] J. Straatsma, G. Bargeman, H.C. van der Horst, J.A. Wesselingh, Can nanofiltration be fully predicted by a model?, J. Membr. Sci., 198 (2002) 273-284.

[7] W. Fang, L. Shi, R. Wang, Mixed polyamide-based composite nanofiltration hollow fiber membranes with improved low-pressure water softening capability, J. Membr. Sci., 468 (2014) 52-61.

[8] G.J. Irvine, S. Rajesh, M. Georgiadis, W.A. Phillip, Ion selective permeation through cellulose acetate membranes in forward osmosis, Environ. Sci. Technol., 47 (2013) 13745-13753.

[9] J. Gilron, N. Gara, O. Kedem, Experimental analysis of negative salt rejection in nanofiltration membranes, J. Membr. Sci., 185 (2001) 223-236.

[10] A.E. Yaroshchuk, Negative rejection of ions in pressure-driven membrane processes, Adv. Colloid Interface Sci., 139 (2008) 150-173.

[11] D.W. Nielsen, G. Jonsson, Bulk-phase criteria for negative ion rejection in nanofiltration of multicomponent salt solutions, Sep. Sci. Technol., 29 (1994) 1165-1182.

[12] R. abu-Rjal, V. Chinaryan, M.Z. Bazant, I. Rubinstein, B. Zaltzman, Effect of concentration polarization on permselectivity, Physical Review E, 89 (2014) 012302.

[13] J.W. Wang, D.S. Dlamini, A.K. Mishra, M.T.M. Pendergast, M.C.Y. Wong, B.B. Mamba, V. Freger, A.R.D. Verliefde, E.M.V. Hoek, A critical review of transport through osmotic membranes, J. Membr. Sci., 454 (2014) 516-537.

[14] S. Bandini, D. Vezzani, Nanofiltration modeling: The role of dielectric exclusion in membrane characterization, Chem. Eng. Sci., 58 (2003) 3303-3326.

[15] W.R. Bowen, A.W. Mohammad, N. Hilal, Characterisation of nanofiltration membranes for predictive purposes - use of salts, uncharged solutes and atomic force microscopy, J. Membr. Sci., 126 (1997) 91-105.

[16] G. Hagmeyer, R. Gimbel, Modelling the salt rejection of nanofiltration membranes for ternary ion mixtures and for single salts at different pH values, Desalination, 117 (1998) 247256.

[17] X. Lefebvre, J. Palmeri, P. David, Nanofiltration theory: An analytic approach for single salts, J. Phys. Chem. B, 108 (2004) 16811-16824.

[18] A. Szymczyk, N. Fatin-Rouge, P. Fievet, C. Ramseyer, A. Vidonne, Identification of dielectric effects in nanofiltration of metallic salts, J. Membr. Sci., 287 (2007) 102-110.

[19] C. Niewersch, C. Abels, R. Li, T. Wintgens, T. Melin, Mass transport modelling to estimate the efficiency of nanofiltration application for the recovery of phosphorus from sewage sludge, Desalination and Water Treatment, 6 (2009) 86-93.

[20] S. Bouranene., P. Fievet, A. Szymczyk, Investigating nanofiltration of multi-ionic solutions using the steric, electric and dielectric exclusion model, Chem. Eng. Sci., 64 (2009) 3789-3798. 
[21] V. Silva, V. Geraldes, A.M. Brites Alves, L. Palacio, P. Prádanos, A. Hernández, Multi-ionic nanofiltration of highly concentrated salt mixtures in the seawater range, Desalination, 277 (2011) 29-39.

[22] C. Mazzoni, S. Bandini, On nanofiltration desal-5 DK performances with calcium chloridewater solutions, Sep. Purif. Tech., 52 (2006) 232-240.

[23] C. Mazzoni, L. Bruni, S. Bandini, Nanofiltration: Role of the electrolyte and pH on desal DK performances, Ind. Eng. Chem. Res., 46 (2007) 2254-2262.

[24] D.S. Dlamini, S. Levchenko, M. Bass, B.B. Mamba, E.M.V. Hoek, J.M. Thwala, V. Freger, Solute hindrance in non-porous membranes: An ATR-FTIR study, Desalination, 368 (2015) 6068.

[25] E. Dražević, K. Košutić, V. Kolev, V. Freger, Does hindered transport theory apply to desalination membranes?, Environ. Sci. Technol., 48 (2014) 11471-11478.

[26] S. Bason, Y. Kaufman, V. Freger, Analysis of ion transport in nanofiltration using phenomenological coefficients and structural characteristics, J. Phys. Chem. B, 114 (2010) 3510-3517.

[27] H. Yan, X.P. Miao, J. Xu, G.Y. Pan, Y. Zhang, Y.T. Shi, M. Guo, Y.Q. Liu, The porous structure of the fully-aromatic polyamide film in reverse osmosis membranes, J. Membr. Sci., 475 (2015) 504-510.

[28] S. Hermans, R. Bernstein, A. Volodin, I.F.J. Vankelecom, Study of synthesis parameters and active layer morphology of interfacially polymerized polyamide-polysulfone membranes,

React. Funct. Polym., 86 (2015) 199-208.

[29] Y.H. La, J. Diep, R. Al-Rasheed, D. Miller, L. Krupp, G.M. Geise, A. Vora, B. Davis, M. Nassar, B.D. Freeman, M. McNeil, G. Dubois, Enhanced desalination performance of polyamide bi-layer membranes prepared by sequential interfacial polymerization, J. Membr. Sci., 437 (2013) 3339.

[30] V. Freger, J. Gilron, S. Belfer, TFC polyamide membranes modified by grafting of hydrophilic polymers: An FT-IR/AFM/TEM study, J. Membr. Sci., 209 (2002) 283-292.

[31] A. Yaroshchuk, M.L. Bruening, E.E. Licon Bernal, Solution-diffusion-electro-migration model and its uses for analysis of nanofiltration, pressure-retarded osmosis and forward osmosis in multi-ionic solutions, J. Membr. Sci., 447 (2013) 463-476.

[32] N. Fridman-Bishop, O. Nir, O. Lahav, V. Freger, Predicting the rejection of major seawater ions by spiral-wound nanofiltration membranes, Environ. Sci. Technol., 49 (2015) 8631-8638.

[33] S. Bason, Y. Oren, V. Freger, Characterization of ion transport in thin films using electrochemical impedance spectroscopy II: Examination of the polyamide layer of RO membranes, J. Membr. Sci., 302 (2007) 10-19.

[34] H. Al-Zoubi, N. Hilal, N.A. Darwish, A.W. Mohammad, Rejection and modelling of sulphate and potassium salts by nanofiltration membranes: Neural network and Spiegler-Kedem model, Desalination, 206 (2007) 42-60.

[35] B. Cuartas-Uribe, M.I. Alcaina-Miranda, E. Soriano-Costa, A. Bes-Piá, Comparison of the behavior of two nanofiltration membranes for sweet whey demineralization, J. Dairy Sci., 90 (2007) 1094-1101.

[36] A. Yaroshchuk, X. Martínez-Lladó, L. Llenas, M. Rovira, J. de Pablo, J. Flores, P. Rubio, Mechanisms of transfer of ionic solutes through composite polymer nano-filtration membranes in view of their high sulfate/chloride selectivities, Desalination and Water Treatment, 6 (2009) 48-53.

[37] N. Pages, A. Yaroshchuk, O. Gibert, J. Luis Cortina, Rejection of trace ionic solutes in nanofiltration: Influence of aqueous phase composition, Chem. Eng. Sci., 104 (2013) 11071115.

[38] A. Yaroshchuk, X. Martínez-Lladó, L. Llenas, M. Rovira, J. de Pablo, Solution-diffusion-film model for the description of pressure-driven trans-membrane transfer of electrolyte mixtures: One dominant salt and trace ions, J. Membr. Sci., 368 (2011) 192-201. 
[39] L. Krasemann, B. Tieke, Selective ion transport across self-assembled alternating multilayers of cationic and anionic polyelectrolytes, Langmuir, 16 (2000) 287-290.

[40] C. Cheng, N. White, H. Shi, M. Robson, M.L. Bruening, Cation separations in electrodialysis through membranes coated with polyelectrolyte multilayers, Polymer, 55 (2014) 1397-1403.

[41] N. White, M. Misovich, E. Alemayehu, A. Yaroshchuk, M.L. Bruening, Highly selective separations of multivalent and monovalent cations in electrodialysis through nafion membranes coated with polyelectrolyte multilayers, Polymer, doi:

10.1016/j.polymer.2015.12.019 (2016).

[42] N. White, M. Misovich, A. Yaroshchuk, M.L. Bruening, Coating of nafion membranes with polyelectrolyte multilayers to achieve high monovalent/divalent cation electrodialysis selectivities, ACS Appl. Mater. Interfaces, 7 (2015) 6620-6628.

[43] K. Maycock, C. Kotzo, F. Muret, Z. Twardowski, J. Ulan, Commercialisation of kvaerner chemetics' sulphate removal system, in: Modern chlor-alkali technology, Blackwell Science Ltd, 2007, pp. 140-151.

[44] G. Bargeman, M. Steensma, A. ten Kate, J.B. Westerink, R.L.M. Demmer, H. Bakkenes, C.F.H. Manuhutu, Nanofiltration as energy-efficient solution for sulfate waste in vacuum salt production, Desalination, 245 (2009) 460-468.

[45] R. Wolters, I. Bettermann, H.-W. Rösler, R. Thomas, Recovery of metals and rinsing water in the tinplate production, Chemie Ingenieur Technik, 84 (2012) 1725-1732.

[46] S. Bason, V. Freger, Phenomenological analysis of transport of mono- and divalent ions in nanofiltration, J. Membr. Sci., 360 (2010) 389-396.

[47] H.J. Cassady, E.C. Cimino, M. Kumar, M.A. Hickner, Specific ion effects on the permselectivity of sulfonated poly(ether sulfone) cation exchange membranes, J. Membr. Sci., 508 (2016) 146-152.

[48] G.M. Geise, H.J. Cassady, D.R. Paul, B.E. Logan, M.A. Hickner, Specific ion effects on membrane potential and the permselectivity of ion exchange membranes, Phys. Chem. Chem. Phys., 16 (2014) 21673-21681.

[49] G.M. Geise, D.R. Paul, B.D. Freeman, Fundamental water and salt transport properties of polymeric materials, Prog. Polymer Sci., 39 (2014) 1-42.

[50] G.M. Geise, L.P. Falcon, B.D. Freeman, D.R. Paul, Sodium chloride sorption in sulfonated polymers for membrane applications, J. Membr. Sci., 423-424 (2012) 195-208.

[51] G.M. Geise, B.D. Freeman, D.R. Paul, Sodium chloride diffusion in sulfonated polymers for membrane applications, J. Membr. Sci., 427 (2013) 186-196.

[52] D.-X. Wang, M. Su, Z.-Y. Yu, X.-L. Wang, M. Ando, T. Shintani, Separation performance of a nanofiltration membrane influenced by species and concentration of ions, Desalination, 175 (2005) 219-225.

[53] V.K. Gupta, S.-T. Hwang, W.B. Krantz, A.R. Greenberg, Characterization of nanofiltration and reverse osmosis membrane performance for aqueous salt solutions using irreversible thermodynamics, Desalination, 208 (2007) 1-18.

[54] M.W. Vonk, J.A.M. Smit, The application of generalized Nernst-Planck equations to the description of ion retention in the hyperfiltration of mixed electrolyte solutions through a neutral membrane, Ber. Bunsen-Ges. Phys. Chem, 88 (1984) 724-732.

[55] M.W. Vonk, J.A.M. Smit, Thermodynamics of ternary systems in reverse osmosis, Desalination, 48 (1983) 105-119.

[56] K. Mehiguene, S. Taha, N. Gondrexon, J. Cabon, G. Dorange, Copper transfer modeling through a nanofiltration membrane in the case of ternary aqueous solution, Desalination, 127 (2000) 135-143.

[57] V. Gekas, B. Hallström, Mass transfer in the membrane concentration polarization layer under turbulent cross flow, J. Membr. Sci., 30 (1987) 153-170.

[58] A. Yaroshchuk, Y. Zhu, M. Bondarenko, M.L. Bruening, Deviations from electroneutrality in membrane barrier layers: A possible mechanism underlying high salt rejections, Langmuir, 32 (2016) 2644-2658. 
[59] D.R. Paul, Reformulation of the solution-diffusion theory of reverse osmosis, J. Membr. Sci., 241 (2004) 371-386.

[60] J.G. Wijmans, R.W. Baker, The solution-diffusion model - a review, J. Membr. Sci., 107 (1995) 1-21.

[61] J. Garcia-Aleman, J.M. Dickson, Permeation of mixed-salt solutions with commercial and pore-filled nanofiltration membranes: Membrane charge inversion phenomena, J. Membr. Sci., 239 (2004) 163-172.

[62] B.W. Stanton, J.J. Harris, M.D. Miller, M.L. Bruening, Ultrathin, multilayered polyelectrolyte films as nanofiltration membranes, Langmuir, 19 (2003) 7038-7042.

[63] Y.W. Ko, R.M. Chen, lon rejection in single and binary mixed electrolyte systems by nanofiltration: Effect of feed concentration, Sep. Sci. Technol., 42 (2007) 3071-3084.

[64] M. Su, D.-X. Wang, X.-L. Wang, M. Ando, T. Shintani, Rejection of ions by NF membranes for binary electrolyte solutions of $\mathrm{NaCl}, \mathrm{NaNO}_{3}, \mathrm{CaCl}_{2}$ and $\mathrm{Ca}\left(\mathrm{NO}_{3}\right)_{2}$, Desalination, 191 (2006) 303-308.

[65] M. Reig, E. Licon, O. Gibert, A. Yaroshchuk, J.L. Cortina, Rejection of ammonium and nitrate from sodium chloride solutions by nanofiltration: Effect of dominant-salt concentration on the trace-ion rejection, Chemical Engineering Journal, 303 (2016) 401-408.

[66] A. Escoda, S. Déon, P. Fievet, Assessment of dielectric contribution in the modeling of multi-ionic transport through nanofiltration membranes, J. Membr. Sci., 378 (2011) 214-223.

[67] S. Déon, A. Escoda, P. Fievet, P. Dutournié, P. Bourseau, How to use a multi-ionic transport model to fully predict rejection of mineral salts by nanofiltration membranes, Chemical Engineering Journal, 189-190 (2012) 24-31. 